\title{
VARIETIES GENERATED BY COMPLETELY 0-SIMPLE SEMIGROUPS
}

\author{
NORMAN R. REILLY
}

(Received 9 December 2005; accepted 9 November 2007)

Communicated by D. Easdown

\begin{abstract}
Kublanovsky has shown that if a subvariety $\mathbf{V}$ of the variety $\mathbf{R S}_{n}$ generated by completely 0 -simple semigroups over groups of exponent $n$ is itself generated by completely 0 -simple semigroups, then it must satisfy one of three conditions: (i) $A_{2} \in \mathbf{V}$; (ii) $N_{1} \notin \mathbf{V}$; (iii) $B_{2} \in \mathbf{V}$ but $A_{0} \notin \mathbf{V}$. The conditions (i) and (ii) are also sufficient conditions. In this note, we complete Kublanovsky's programme by refining condition (iii) to obtain a complete set of conditions that are both necessary and sufficient.
\end{abstract}

2000 Mathematics subject classification: 20M07, $08 \mathrm{~B} 15$.

Keywords and phrases: semigroup, variety, Rees-Sushkevich varieties, exact, completely 0-simple.

\section{Introduction and background}

The lattice of varieties of semigroups is a fascinating structure, many parts of which have been intensively studied. Completely 0-simple semigroups are one of the basic building blocks for semigroups, especially finite semigroups, and so the varieties that they generate deserve special attention.

Following the necessary background material in this section, we describe Kublanovsky's results concerning exact varieties, that is, varieties that are generated by completely 0 -simple semigroups over groups of exponent dividing $n$. Kublanovsky's proof of the sufficiency of certain conditions leads us to the variety defined by the identity $x^{n} y^{n}=y^{n} x^{n}$, which can be characterized as the largest variety not containing any of the semigroups $L_{2}, R_{2}, A_{0}$. As preparation, we also characterize the variety defined by the identity $x^{n} y^{n}=\left(x^{n} y^{n}\right)^{n+1}$. In Section 3 we introduce a construction that is used in Section 4 to show that the mapping $\mathbf{V} \longmapsto \mathbf{V} \cap \mathbf{G}_{n}$ is a complete retraction of the lattice $\mathcal{L}\left(\mathbf{R S}_{n}\right)$ of Rees-Sushkevich varieties to the lattice $\mathcal{L}\left(\mathbf{G}_{n}\right)$ of varieties of groups of exponent dividing $n$.

This work was supported by NSERC grant A4044.

(C) 2008 Australian Mathematical Society 1446-7887/08 \$A2.00+0.00 
In Sections 5 and 6 we show that any variety in the interval $\left[\mathbf{B}_{\mathbf{2}}, \mathbf{N B}_{\mathbf{2}} \vee \mathbf{G}_{n}\right]$ is the join of the largest group variety and the largest aperiodic variety that it contains. We are then able to show in Section 7 that the exact varieties do not form a sublattice of $\mathcal{L}\left(\mathbf{R S}_{n}\right)$ and that the interval $\left[\mathbf{B}_{2}, \mathbf{N B}_{\mathbf{2}} \vee \mathbf{G}_{n}\right]$ is the largest interval of the form $\left[\mathbf{B}_{2}, \mathbf{V}\right]$ consisting entirely of exact varieties. For each prime $p$, we introduce a $2 \times 2$ nonorthodox completely simple semigroup $M_{p}$ over the cyclic group of order $p$. Denoting by $\mathbf{M}_{p}$ the variety generated by $M_{p}$, we show in Section 8 that, for each prime $p$ dividing $n$, the interval $\left[\mathbf{B}_{\mathbf{2}} \vee \mathbf{M}_{p},\left[x^{n} y^{n}=\left(x^{n} y^{n}\right)^{n+1}\right] \cap \mathbf{R S}_{n}\right]$ consists entirely of exact varieties. In this way we can give a complete characterization of the exact subvarieties of $\mathbf{R S}_{n}$ and describe them as consisting of a finite set of intervals.

The following semigroups defined by generators and relations will be important to our discussions:

$$
\begin{aligned}
N_{1} & =\left\langle a, 0 \mid a^{2}=0\right\rangle, \\
Y_{2} & =\left\langle a, 0 \mid a^{2}=a\right\rangle, \\
A_{0} & =\left\langle a, b \mid a^{2}=a, b^{2}=b, b a=0\right\rangle, \\
L_{2} & =\left\langle e, f \mid e^{2}=e=e f, f^{2}=f=f e\right\rangle, \\
R_{2} & =\left\langle e, f \mid e^{2}=e=f e, f^{2}=f=e f\right\rangle .
\end{aligned}
$$

The following Rees-matrix semigroups will also be important:

$$
\begin{aligned}
& A_{2}=\mathcal{M}^{0}\left(\{0,1\},\{1\},\{0,1\} ; P_{2}\right), \\
& B_{2}=\mathcal{M}^{0}\left(\{0,1\},\{1\},\{0,1\} ; I_{2}\right),
\end{aligned}
$$

where

$$
P_{2}=\left[\begin{array}{ll}
1 & 1 \\
1 & 0
\end{array}\right], \quad I_{2}=\left[\begin{array}{ll}
1 & 0 \\
0 & 1
\end{array}\right] .
$$

Alternatively, we may describe $B_{2}$ by generators and relations:

$$
B_{2}=\left\langle a, b, 0 \mid a b a=a, b a b=b, a^{2}=b^{2}=0\right\rangle .
$$

For any semigroup $S$ (respectively, family of semigroups $\left\{S_{\alpha} \mid \alpha \in A\right\}$ ) we denote by $V(S)$ (respectively, $V\left(\left\{S_{\alpha} \mid \alpha \in A\right\}\right)$ ) the variety generated by $S$ (respectively, the semigroups $\left.S_{\alpha}, \alpha \in A\right)$. We write $\mathbf{V}=\left[u_{1}=v_{1}, \ldots, u_{m}=v_{m}\right]$ for the semigroup variety defined by the identities $u_{1}=v_{1}, \ldots, u_{m}=v_{m}$. For any variety $\mathbf{V}$ we denote the lattice of subvarieties of $\mathbf{V}$ by $\mathcal{L}(\mathbf{V})$. We adopt the notation:

$$
\begin{aligned}
\mathbf{L Z} & =V\left(L_{2}\right), \quad \mathbf{R Z}=V\left(R_{2}\right), \\
\mathbf{A}_{\mathbf{2}}^{*} & =V\left(A_{2}\right), \quad \mathbf{B}_{\mathbf{2}}=V\left(B_{2}\right), \\
\mathbf{L N B}_{\mathbf{2}} & =\mathbf{B}_{\mathbf{2}} \vee \mathbf{L Z}, \quad \mathbf{R} \mathbf{N B}_{\mathbf{2}}=\mathbf{B}_{\mathbf{2}} \vee \mathbf{R Z}, \\
\mathbf{N B}_{\mathbf{2}} & =\mathbf{B}_{\mathbf{2}} \vee \mathbf{L Z} \vee \mathbf{R Z}, \\
\mathbf{G}_{n} & =\text { variety of groups of exponent dividing } n .
\end{aligned}
$$

In the next lemma we present bases of identities for several varieties that are critical to our discussions. 
LEMMA 1.1. The following hold:

(i) $\quad \mathbf{B}_{\mathbf{2}}=\left[x^{2}=x^{3}, x y x=x y x y x, x^{2} y^{2}=y^{2} x^{2}\right]$.

(ii) $\quad \mathbf{L N B}_{2}=\left[x^{2}=x^{3}, x y x=x y x y x, a x^{2} y^{2}=a y^{2} x^{2}\right]$.

(iii) $\mathbf{R N B}_{\mathbf{2}}=\left[x^{2}=x^{3}, x y x=x y x y x, x^{2} y^{2} b=y^{2} x^{2} b\right]$.

(iv) $\mathbf{N B}_{\mathbf{2}}=\left[x^{2}=x^{3}, x y x=x y x y x, a x^{2} y^{2} b=a y^{2} x^{2} b\right]$.

(v) $\quad \mathbf{A}_{2}^{*}=\left[x^{2}=x^{3}, x y x=x y x y x, x y x z x=x z x y x\right]$.

PROOF. (i) Trahtman [11] was the first to present a basis of identities for $\mathbf{B}_{\mathbf{2}}$. His proof contains a small lacuna, which can be fixed, however, in a number of ways, one of which can be found in [9, Theorem 7.4].

(ii, iii, iv) These follow in a straightforward way from (i). Details can be found in [9].

(v) See Trahtman [12].

Let $X=\left\{x_{1}, x_{2}, \ldots\right\}$ be a countably-infinite alphabet, and let $X^{+}$(respectively $X^{*}$ ) denote the free semigroup (respectively, free monoid) on the set $X$. For any word $w \in X^{+}$, let

$$
\begin{aligned}
c(w) & =\text { set of letters appearing in } w \\
|w| & =\text { the number of letters in } w, \text { counting repetitions, } \\
h(w) & =\text { the first letter in } w \\
t(w) & =\text { the last letter in } w
\end{aligned}
$$

For $u \in X^{+}$, let $e(u)$ denote the set of all subwords of $u$ of length 2 . For any finite subset $A$ of $X^{2}$, let

$$
\kappa(A)=A \cup\{x y \mid \exists a, b, x, y \in X \text { with } a b, a y, x b \in A\},
$$

and define $\kappa^{n}(A)$ inductively by:

$$
\begin{aligned}
\kappa^{0}(A) & =A, \\
\kappa^{n+1}(A) & =\kappa\left(\kappa^{n}(A)\right) .
\end{aligned}
$$

Intuitively, we think of $\kappa$ as 'completing' squares:

\begin{tabular}{|c|c|c|}
\hline & $b$ & $y$ \\
\hline$a$ & $\checkmark$ & $\checkmark$ \\
\hline$x$ & $\checkmark$ & \\
\hline
\end{tabular}

For finite $A$, there must exist an integer $n$ with $\kappa^{n+1}(A)=\kappa^{n}(A)$. When $u \in X^{+}$, $A=e(u)$ and $n$ such that $\kappa^{n+1}(e(u))=\kappa^{n}(e(u))$, we define

$$
E(u)=\kappa^{n}(u) .
$$

For any $x \in c(u), u \in X^{+}$, let

$$
\begin{aligned}
& L(x)=\{p \in X: p x \in E(u)\}, \\
& R(x)=\{p \in X: x p \in E(u)\} .
\end{aligned}
$$


Since $B_{2}$ and $B_{2} \times L_{2} \times R_{2}$ are finite semigroups, their word problems are 'trivially' solvable by considering all possible substitutions of variables into these semigroups. However, such a computation is exponential in the number of variables, which is neither very practical, nor very useful theoretically. The solutions in the next lemma are both polynomial and theoretically applicable.

LEMMA 1.2. The following hold:

(i) For $u, v \in X^{+}, \mathbf{B}_{2}$ satisfies the identity $u=v$ if and only if the following conditions are satisfied:

$$
\begin{gathered}
c(u)=c(v), \quad E(u)=E(v), \\
\text { either } \quad h(u)=h(v) \quad \text { or } \quad L(h(u)) \cap L(h(v)) \neq \emptyset, \\
\text { either } \quad t(u)=t(v) \quad \text { or } \quad R(t(u)) \cap R(t(v)) \neq \emptyset .
\end{gathered}
$$

(ii) For $u, v \in X^{+}, \mathbf{N B}_{2}$ satisfies the identity $u=v$ if and only if the following conditions are satisfied:

$$
c(u)=c(v), \quad h(u)=h(v), \quad t(u)=t(v) \quad \text { and } \quad E(u)=E(v) .
$$

PROOF. (i) This solution to the word problem for $\mathbf{B}_{\mathbf{2}}$ can be found in Reilly [9]. It can also be derived from the somewhat different solution provided by Mashevitzky [6].

(ii) This can be derived from part (i), and can also be found in Reilly [9].

The following observation regarding the identities satisfied by $\mathbf{B}_{\mathbf{2}}$ is easy to verify directly, or can be found in [9, Corollary 6.3].

LEMMA 1.3. Let $s, t \in X$ and $u, v \in X^{+}$be such that $s, t \notin c(u)=c(v)$. If $\mathbf{B}_{2}$ satisfies the identity sut $=s v t$, then $\mathbf{B}_{2}$ also satisfies the identity $u=v$.

We denote by $\mathbf{C S}_{n}^{\mathbf{0}}$ the class of completely 0-simple semigroups over groups of exponent dividing $n$ and we denote by $\mathbf{R S}_{n}$ the variety generated by all completely 0 -simple semigroups over groups of exponent dividing $n$. We call any subvariety of $\mathbf{R S}_{n}$ a Rees-Sushkevich variety. For the sake of simplicity we will interpret 'completely 0 -simple' to mean either completely 0 -simple or completely simple, as the occasion requires.

THEOREM 1.4 (Hall et al. [2, Proposition 3.3]). The following set of identities is a basis of identities for $\mathbf{R S}_{n}$ :

$$
\begin{gathered}
x^{n+2}=x^{2}, \\
(x y)^{n+1} x=x y x, \\
x y x(z x)^{n}=x(z x)^{n} y x .
\end{gathered}
$$

If we take $n=1$ in Theorem 1.4 , then the identities $\left(\mathrm{I}_{n}\right),\left(\mathrm{II}_{n}\right)$ and $\left(\mathrm{III}_{n}\right)$ reduce to exactly the basis for $\mathbf{A}_{2}^{*}$ given in Lemma 1.1(v). Thus we have the following. 
Corollary 1.5. $\mathbf{A}_{\mathbf{2}}^{*}=\mathbf{R S}_{\mathbf{1}}$.

A word $w \in X^{+}$is said to be covered by cycles if, for each $x_{i} x_{j} \in e(w)$, there exists a subword $u$ of $w$ with $x_{i} x_{j} \in e(u)$ and $h(u)=t(u)$. An identity $u=v\left(u, v \in X^{+}\right)$is said to be covered by cycles if $u$ and $v$ are both covered by cycles.

Lemma 1.6 (Mashevitzky [7, Lemma 6]; corrected in Volkov [13]). Let $w \in X^{+}$be covered by cycles and $\theta: X^{+} \rightarrow S \in \mathbf{R S}_{n}$ be any homomorphism. Then $\theta(w)$ is a regular element of $S$.

A useful fact concerning regular elements is contained in the following.

LEMMA 1.7 (Hall et al. [2, Lemma 3.2]). Let $S \in \mathbf{R S}_{n}$. For any distinct regular elements $a, b \in S$, there exists a completely 0 -simple semigroup $K$ and a surjective homomorphism $\varphi: S \rightarrow K$ such that $\varphi(a) \neq \varphi(b)$.

A word $u \in X^{+}$is said to be a repeated word if each of its letters either appears in it at least twice or is contained in a subword starting and ending with the same letter.

LEMMA 1.8. Let $u \in X^{+}$be a repeated word and $\rho$ be a fully invariant congruence on $X^{+}$such that $X^{+} / \rho$ satisfies the identity

$$
x y x=x(y x)^{n+1} \quad(n>1) .
$$

Then $u$ is $\rho$-equivalent to a word that is a product words covered by cycles. In particular, it is $\rho$-equivalent to a word of the form

$$
x_{1} u_{1} x_{1} x_{2} u_{2} x_{2} \ldots x_{m} u_{m} x_{m}
$$

where $x_{i} \in X, u_{i} \in X^{*}$.

ProOF. See Reilly [9, Lemma 5.1(ii)].

Throughout we take advantage of the Rees theorem representing completely 0 -simple semigroups as regular Rees matrix semigroups, for which we adopt the notation $\mathcal{M}^{0}(I, G, \Lambda ; P)$ from [8]. Whenever we write $S=\mathcal{M}^{0}(I, G, \Lambda ; P)$ we intend that the matrix $P=\left(p_{\lambda i}\right)$ be regular, that is,

$$
\begin{array}{r}
(\forall i \in I)(\exists \lambda \in \Lambda) \quad p_{\lambda i} \neq 0 \quad \text { and } \\
(\forall \lambda \in \Lambda)(\exists i \in I) \quad p_{\lambda i} \neq 0 .
\end{array}
$$

We refer the reader to Howie [3] for information on this and other basic aspects of semigroup theory.

\section{Basic results}

In [4], Kublanovsky provides a wealth of information on Rees-Sushkevich varieties. One fascinating idea introduced there was that it might be possible to give a simple characterization of the Rees-Sushkevich varieties that are actually generated by completely 0 -simple semigroups by means of the inclusion or exclusion of some small family of semigroups. 
Theorem 2.1 (Kublanovsky et al. [5]). Let $\mathbf{V} \in \mathcal{L}\left(\mathbf{R S}_{n}\right)$. If $\mathbf{V}$ is generated by completely 0-simple semigroups, then one of the following conditions must hold:

(1) $A_{2} \in \mathbf{V}$;

(2) $N_{1} \notin \mathbf{V}$;

(3) $B_{2} \in \mathbf{V}, A_{0} \notin \mathbf{V}$.

Conversely, if $\mathbf{V}$ satisfies condition (1) or (2) or the condition

(3') $B_{2} \in \mathbf{V}, A_{0}, L_{2}, R_{2} \notin \mathbf{V}$,

then $\mathbf{V}$ is generated by completely 0 -simple semigroups.

In subsequent sections, we will show that condition $\left(3^{\prime}\right)$ is equivalent to saying that the interval $\left[\mathbf{B}_{\mathbf{2}}, \mathbf{B}_{\mathbf{2}} \vee \mathbf{G}_{n}\right]$ consists of exact varieties. We will then extend this result to show that the interval $\left[\mathbf{B}_{\mathbf{2}}, \mathbf{N B}_{\mathbf{2}} \vee \mathbf{G}_{n}\right]$ consists of exact varieties and that is the largest possible such interval, though it does not capture all the remaining exact varieties.

We now provide some preliminary observations regarding condition $\left(3^{\prime}\right)$.

Lemma 2.2. Let $S \in \mathbf{R S}_{n}, n>1$. Then the following statements are equivalent.

(i) S satisfies the identity $x^{n} y^{n}=y^{n} x^{n}$.

(ii) $S$ is not divisible by $A_{0}, L_{2}$ or $R_{2}$.

We can now characterize in terms of the lattice of Rees-Sushkevich varieties the applicability of condition (iii) in Theorem 2.1.

Lemma 2.3. Let $\mathbf{V}$ be a Rees-Sushkevich variety. Then the following statements are equivalent:

(i) $\quad \mathbf{B}_{2} \subseteq \mathbf{V}$ and $A_{0}, L_{2}, R_{2} \notin \mathbf{V}$;

(ii) $\mathbf{V} \in\left[\mathbf{B}_{2},\left[\mathrm{I}_{n}, \mathrm{II}_{n}, \mathrm{III}_{n}, x^{n} y^{n}=y^{n} x^{n}\right]\right]$;

(iii) $\mathbf{V} \cap \mathbf{A}_{\mathbf{2}}^{*}=\mathbf{B}_{\mathbf{2}}$.

Proof. It follows immediately from Lemma 2.2 that (i) implies (ii).

To show that (ii) implies (iii):

$$
\begin{aligned}
\mathbf{B}_{2} & \subseteq \mathbf{V} \cap \mathbf{A}_{\mathbf{2}}^{*} \\
& \subseteq\left[x^{n} y^{n}=y^{n} x^{n}\right] \cap \mathbf{A}_{\mathbf{2}}^{*} \\
& =\mathbf{B}_{\mathbf{2}} \quad \text { by Lemma 1.1(i), }
\end{aligned}
$$

whence $\mathbf{V} \cap \mathbf{A}_{\mathbf{2}}^{*}=\mathbf{B}_{\mathbf{2}}$.

From the fact that $A_{0}, L_{2}, R_{2}$ belong to $\mathbf{A}_{\mathbf{2}}^{*}$ but not $\mathbf{B}_{2}$, it follows immediately that (iii) implies (i).

COROLlaRY 2.4. All varieties in the interval $\left[\mathbf{B}_{\mathbf{2}},\left[\mathrm{I}_{n}, \mathrm{II}_{n}, \mathrm{III}_{n}, x^{n} y^{n}=y^{n} x^{n}\right]\right]$ are exact.

PROOF. This follows immediately from Theorem 2.1 and Lemma 2.3. 
We conclude this section with two useful characterizations of the variety given by the identities in Corollary 2.4.

COROllary 2.5. Let

$$
\mathbf{V}=\left[\mathrm{I}_{n}, \mathrm{II}_{n}, \mathrm{III}_{n}, x^{n} y^{n}=y^{n} x^{n}\right] \quad(n>1) .
$$

(i) $\quad \mathbf{V}$ is the largest subvariety of $\mathbf{R S}_{n}$ such that $\mathbf{V} \cap \mathbf{R S}_{\mathbf{1}}=\mathbf{B}_{\mathbf{2}}$.

(ii) $\mathbf{V}$ is the variety generated by all Brandt semigroups over groups with exponent dividing $n$.

Proof. (i) Clearly $\mathbf{B}_{\mathbf{2}} \subseteq \mathbf{V} \cap \mathbf{R S}_{\mathbf{1}}$. Moreover, in combination with $x^{2}=x^{3}$ the identity $x^{n} y^{n}=y^{n} x^{n}$ reduces to $x^{2} y^{2}=y^{2} x^{2}$. Thus $\mathbf{V} \cap \mathbf{R S}_{\mathbf{1}} \subseteq \mathbf{B}_{\mathbf{2}}$ and equality prevails.

If $S \in \mathbf{R S}_{n} \backslash \mathbf{V}$, then $S$ does not satisfy the identity $x^{n} y^{n}=y^{n} x^{n}$, so that, by Lemma 2.2, $V(S)$ contains $L_{2}, R_{2}$ or $A_{0}$. Thus $V(S) \cap \mathbf{R S}_{\mathbf{1}} \nsubseteq \mathbf{B}_{\mathbf{2}}$. Therefore $\mathbf{V}$ is the largest such variety.

(ii) See [2, Proposition 3.9].

In Corollary 5.3 (below), we will have a further characterization of the variety discussed in Corollary 2.5 as

$$
\mathbf{V}=\mathbf{B}_{2} \vee \mathbf{G}_{n} .
$$

The condition that $A_{0}$ not belong to a subvariety of $\mathbf{R S}_{n}$ is an important one, for reasons brought out in the next result. Recall that an element $a \in S$ is said to be regular if there exists an element $x \in S$ with $a=a x a$. For any semigroup $S$, let

$$
\operatorname{Reg}(S)=\{a \in S \mid a \text { is regular }\} .
$$

Proposition 2.6. Let $\mathbf{V} \in \mathcal{L}\left(\mathbf{R S}_{n}\right), n>1$. Then the following statements are equivalent.

(i) V satisfies the identity $x^{n} y^{n}=\left(x^{n} y^{n}\right)^{n+1}$.

(ii) For all $S \in \mathbf{V}, \operatorname{Reg}(S)$ is a subsemigroup.

(iii) $A_{0} \notin \mathbf{V}$.

Proof. To show that (i) implies (ii): let $a, b \in \operatorname{Reg}(S)$, where $S \in \mathbf{V}$. Then there exist elements $a^{\prime}, b^{\prime} \in S$ with $a=a a^{\prime} a, b=b b^{\prime} b$. The elements $a^{\prime} a$ and $b b^{\prime}$ are idempotents, and so we may write

$$
\begin{aligned}
a b\left[b^{\prime}\left(a^{\prime} a b b^{\prime}\right)^{n-1} a^{\prime}\right] a b & =a a^{\prime} a b\left[b^{\prime}\left(a^{\prime} a b b^{\prime}\right)^{n-1} a^{\prime}\right] a b b^{\prime} b \\
& =a\left(a^{\prime} a b b^{\prime}\right)^{n+1} b \\
& =a\left(\left(a^{\prime} a\right)^{n}\left(b b^{\prime}\right)^{n}\right)^{n+1} b \\
& =a\left(a^{\prime} a\right)^{n}\left(b b^{\prime}\right)^{n} b \\
& =a b .
\end{aligned}
$$

Thus $a b \in \operatorname{Reg}(S)$ and (ii) holds. 
To show that (ii) implies (i): let $x, y \in S \in \mathbf{V}$. By the identity $\mathrm{I}_{n}, x^{n}$ and $y^{n}$ are idempotents, whence $x^{n}, y^{n} \in \operatorname{Reg}(S)$. Now $\left\langle x^{n}, y^{n}\right\rangle$ is a subsemigroup of $S$, and so belongs to $\mathbf{V}$. Hence, by hypothesis, $\operatorname{Reg}\left(\left\langle x^{n}, y^{n}\right\rangle\right)$ is a subsemigroup of $\left\langle x^{n}, y^{n}\right\rangle$. Since $x^{n}, y^{n} \in \operatorname{Reg}\left(\left\langle x^{n}, y^{n}\right\rangle\right)$, it follows that $x^{n} y^{n} \in \operatorname{Reg}\left(\left\langle x^{n}, y^{n}\right\rangle\right)$. Therefore there exists an element $z \in\left\langle x^{n}, y^{n}\right\rangle$ with

$$
x^{n} y^{n} z x^{n} y^{n}=x^{n} y^{n},
$$

which implies that

$$
x^{n} y^{n}=\left(x^{n} y^{n}\right)^{k},
$$

for some integer $k>1$. Thus $\left\langle x^{n} y^{n}\right\rangle$ is a subgroup of $\left\langle x^{n}, y^{n}\right\rangle$ and of $S$ and so is of exponent dividing $n$. Hence we must have $\left(x^{n} y^{n}\right)^{n+1}=x^{n} y^{n}$, and (i) holds.

To show that (i) implies (iii): as $A_{0}$ does not satisfy the identity $x^{n} y^{n}=\left(x^{n} y^{n}\right)^{n+1}$, it is evident that $A_{0}$ cannot belong to $\mathbf{V}$.

To show that (iii) implies (i): suppose that $\mathbf{V}$ does not satisfy the identity $x^{n} y^{n}=\left(x^{n} y^{n}\right)^{n+1}$. Then there exist $S \in \mathbf{V}$ and $x, y \in S$ such that $x^{n} y^{n} \neq\left(x^{n} y^{n}\right)^{n+1}$. Let $e=x^{n}, f=y^{n}$. Since $S \in \mathbf{R S}_{n}$, we know that $e$ and $f$ are idempotents. Let

$$
T=\langle e, f\rangle, \quad I=T f e T .
$$

Then $I$ is an ideal of $T$. Suppose that $e f \in I$. Then there exist $a, b \in T$ with $e f=a f e b$. Hence, for some $k>1$, we have $e f=(e f)^{k}$. But that means that $\langle e f\rangle$ is a cyclic subgroup of $T$ and must have order dividing $n$, so that

$$
\left(x^{n} y^{n}\right)^{n+1}=(e f)^{n+1}=e f=x^{n} y^{n},
$$

contradicting our assumption. Hence ef $\notin I$ and $T / I=\{e, f$, ef, $I\} \cong A_{0}$. Thus $A_{0} \in \mathbf{V}$, which is again a contradiction. Hence $\mathbf{V}$ must satisfy the identity in (i).

\section{Construction of a cover}

In this section, we wish to construct a suitable cover for Rees matrix semigroups that satisfy the identity $a x^{n} y^{n} b=a y^{n} x^{n} b$.

LEMMA 3.1. Let $S=\mathcal{M}^{0}(I, G, \Lambda ; P)$ satisfy the identity $a x^{n} y^{n} b=a y^{n} x^{n} b$. Then the following hold.

(i) There exists a $\{0,1\}$-matrix $Q=\left(q_{\lambda i}\right)$ such that $S \cong \mathcal{M}^{0}(I, G, \Lambda ; Q)$.

(ii) There exists a set $A$ and partitions $I=\bigcup_{\alpha \in A} I_{\alpha}, \Lambda=\bigcup_{\alpha \in A} \Lambda_{\alpha}$ of $I$ and $\Lambda$ such that

$$
q_{\lambda i} \neq 0 \Longleftrightarrow \text { there exits } \alpha \in A \text { with } i \in I_{\alpha} \text { and } \lambda \in \Lambda_{\alpha} \text {. }
$$

Proof. Part (i) follows from Graham [1, Corollary 2], and part (ii) follows from Reilly [10, Theorem 7.2(xiv)]. 
Let $S, T$ be semigroups, and let $K$ be an ideal of $T$. We say that $T$ is a retract ideal extension of $K$ by $S$ if:

(a) $T / K \cong S$; and

(b) there exists an endomorphism $\varphi: T \rightarrow K$ with $\varphi(k)=k$ for all $k \in K$.

Let $S=\mathcal{M}^{0}(I, G, \Lambda ; P)$, where:

(i) $G \in \mathbf{G}_{n}$ and $G$ is nontrivial;

(ii) $S$ satisfies the identity $a x^{n} y^{n} b=a y^{n} x^{n} b$;

(iii) $P$ is a $\{0,1\}$-matrix.

Let $I=\bigcup_{\alpha \in A} I_{\alpha}, \quad \Lambda=\bigcup_{\alpha \in A} \Lambda_{\alpha}$ be partitions of $I$ and $\Lambda$ such that

$$
p_{\lambda i} \neq 0 \Longleftrightarrow(\exists \alpha \in A) i \in I_{\alpha}, \quad \lambda \in \Lambda_{\alpha}
$$

Let $K \in V(G) \cap \mathbf{G}_{n}$ be such that $G$ is a subgroup of $K$, and let $i \mapsto u_{i}$ be a mapping of $I$ into $K$ such that:

$\mathrm{K}(\mathrm{i}) u_{i}=u_{j} \Longleftrightarrow(\exists \alpha \in A) i, j \in I_{\alpha}$;

$\mathrm{K}(\mathrm{ii})\left\{u_{i} u_{j}^{-1} \mid i, j \in I, u_{i} \neq u_{j}\right\} \cap G=\emptyset$.

Let $\lambda \mapsto u_{\lambda} \in K$ be defined by setting $u_{\lambda}=u_{i}$ where $i \in I_{\alpha}, \lambda \in \Lambda_{\alpha}$.

Let $S^{*}=S \backslash\{0\}$ and $T=S^{*} \cup K$, and define multiplication in $T$ as follows:

$$
\begin{gathered}
(i, g, \lambda)(j, h, \mu)=u_{i}^{-1} g u_{\lambda} u_{j}^{-1} h u_{\mu} \quad\left(p_{\lambda j}=0\right), \\
(i, g, \lambda) k=u_{i}^{-1} g u_{\lambda} k, \quad k(i, g, \lambda)=k u_{i}^{-1} g u_{\lambda},
\end{gathered}
$$

with multiplication within $S^{*}$ and $K$ as given.

THEOREM 3.2. With $S, K$ and $T$ as above:

(i) the mapping

$$
\chi:(i, g, \lambda) \longmapsto u_{i}^{-1} g u_{\lambda}
$$

is a partial homomorphism of $S^{*}$ into $K$;

(ii) $T$ is a retract ideal extension of $K$ by $S$, with retraction $\zeta: T \rightarrow K$ defined by

$$
\begin{aligned}
\zeta((i, g, \lambda)) & =\chi((i, g, \lambda)) \quad\left((i, g, \lambda) \in S^{*}\right), \\
\zeta(k) & =k \quad(k \in K)
\end{aligned}
$$

(iii) $\chi$ is injective on each $\mathcal{H}$-class of $T$;

(iv) $S \cong T / K$;

(v) $\mathcal{H}$ is a congruence on $S$ and $T$ and

$$
T / \mathcal{H} \cong S / \mathcal{H} \in \mathbf{N B}_{2}
$$

(vi) $T$ is isomorphic to a subsemigroup of $K \times S / \mathcal{H}$. 
Proof. (i) For $p_{\lambda j} \neq 0$, we have $p_{\lambda j}=1$ and

$$
\begin{aligned}
\chi((i, g, \lambda)(j, h, \mu)) & =\chi(i, g h, \mu) \\
& =u_{i}^{-1} g h u_{\mu} .
\end{aligned}
$$

Since $p_{\lambda j} \neq 0$, there exists $\alpha \in A$ with $j \in I_{\alpha}, \lambda \in \Lambda_{\alpha}$ such that $u_{\lambda}=u_{j}$ and

$$
\begin{aligned}
\chi(i, g, \lambda) \chi(j, h, \mu) & =u_{i}^{-1} g u_{\lambda} u_{j}^{-1} h u_{\mu} \\
& =u_{i}^{-1} g h u_{\mu},
\end{aligned}
$$

as required.

(ii) See Petrich and Reilly [8, Lemma I.6.3].

(iii) The $\mathcal{H}$-classes of $T$ are $K$ and the nonzero $\mathcal{H}$-classes of $S$. By definition, the restriction of $\zeta$ to $K$ is the identity mapping. Consider any pair $(i, g, \lambda),(i, h, \lambda)$ of $\mathcal{H}$-related elements in $S^{*}$. We have

$$
\begin{aligned}
\zeta(i, g, \lambda)=\zeta(i, h, \lambda) & \Longrightarrow u_{i}^{-1} g u_{\lambda}=u_{i}^{-1} h u_{\lambda} \\
& \Longrightarrow g=h \\
& \Longrightarrow(i, g, \lambda)=(i, h, \lambda) .
\end{aligned}
$$

Thus $\zeta$ is injective on all $\mathcal{H}$-classes.

(iv) This follows immediately from (ii).

(v) This follows from the observation that the $\mathcal{H}$-classes of $T$ are the nonzero $\mathcal{H}$ classes of $S$ together with $K$.

Now $S / \mathcal{H}$ is aperiodic, and so it satisfies the identity $x^{2}=x^{3}$. In conjunction with the identity $a x^{n} y^{n} b=a y^{n} x^{n} b$, this yields the identity $a x^{2} y^{2} b=a y^{2} x^{2} b$. Hence, by Lemma 1.1, $S / \mathcal{H} \in \mathbf{N B}_{2}$.

(vi) Define $\varphi: T \rightarrow K \times T / \mathcal{H}$ by

$$
\varphi(t)=\left(\zeta(t), H_{t}\right)
$$

The mapping $\zeta$ is a retraction (and so a homomorphism) of $T$ to $K$, while the mapping $t \mapsto H_{t}$ is a homomorphism since $\mathcal{H}$ is a congruence. Hence $\varphi$ is a homomorphism. The homomorphism $t \mapsto H_{t}$ separates $\mathcal{H}$-classes of $T$, while the homomorphism $\zeta: T \rightarrow K$ is, by part (iii), injective when restricted to any $\mathcal{H}$-class. Therefore $\varphi$ is a monomorphism. The claim now follows from part (v).

\section{A complete homomorphism}

Clearly the groups contained in $\mathbf{R} \mathbf{S}_{n}$ comprise the variety $\mathbf{G}_{n}$, and so it comes as no surprise that the variety $\mathbf{G}_{n}$ of groups of exponent dividing $n$ figures prominently in the study of $\mathbf{R S}_{n}$. In this section we will show that the mapping $\mathbf{V} \longmapsto \mathbf{V} \cap \mathbf{G}_{n}$ determines a complete retraction of $\mathcal{L}\left(\mathbf{R S}_{n}\right)$ to $\mathcal{L}\left(\mathbf{G}_{n}\right)$. 
For any $x \in G \in \mathbf{G}_{n}$, we have $x^{-1}=x^{n-1}$, and so it follows that every (group) subvariety of $\mathbf{G}_{n}$ can be defined by identities of the form

$$
w y=y w=y,
$$

where $w \in X^{+}$and $y \in X$.

Let $u=u\left(x_{1}, \ldots, x_{m}\right)$ be a word in the variables $x_{1}, \ldots, x_{m}$. We wish to define an associated word. First let $w$ denote any word that contains as subwords all products $x_{i} x_{j}$ of all pairs of the variables $x_{1}, \ldots, x_{m}$ and let $x_{0}$ be a variable distinct from $x_{1}, \ldots, x_{m}$. Now define

$$
x_{i}^{\gamma}=\left(x_{0} w x_{0}\right)^{n} x_{0}^{n} x_{i} x_{0}^{n} \quad(1 \leq i \leq m),
$$

and

$$
u^{\gamma}\left(x_{0}, x_{1}, \ldots, x_{m}\right)=u\left(x_{1}^{\gamma}, x_{2}^{\gamma}, \ldots, x_{m}^{\gamma}\right) .
$$

LEMMA 4.1. Let $F=F\left(x_{0}, x_{1}, \ldots, x_{n}\right)$ be the free semigroup in $\mathbf{R S}_{n}$ on $x_{0}, x_{1}, \ldots, x_{m}$. Then the elements $x_{i}^{\gamma}, i=1, \ldots, n$, generate a subgroup of $F$.

PROOF. Since $\mathbf{R S}_{n}$ is generated by completely 0-simple semigroups, there exist $S_{\alpha} \in \mathbf{C S}^{\mathbf{0}}{ }_{n}, \alpha \in A$ such that $F$ is (isomorphic to) a subsemigroup of $\prod_{\alpha \in A} S_{\alpha}$; that is,

$$
F \leq \prod_{\alpha \in A} S_{\alpha}
$$

Let $\alpha \in A$. If there exist $i, j$ such that $x_{i}(\alpha) x_{j}(\alpha)=0$, then $w(\alpha)=0$, so that $x_{i}^{\gamma}(\alpha)=0$ for all $i$. On the other hand, if $x_{i}(\alpha) x_{j}(\alpha) \neq 0$ for all $i, j$ with $0 \leq i, j \leq m$, then $\left\langle\left\{x_{i}(\alpha)\right\}\right\rangle$ is a completely simple subsemigroup of $S_{\alpha}$. Consequently, $x_{i}^{\gamma}(\alpha)$ $\in H_{x_{0}(\alpha)}=T_{\alpha}$ for all $i$ with $1 \leq i \leq m$, where $T_{\alpha}$ is a subgroup of $S_{\alpha}$. Hence

$$
\left\langle\left\{x_{i}^{\gamma}\right\}\right\rangle \leq \prod_{\alpha \in A} T_{\alpha},
$$

where each $T_{\alpha}$ is a subgroup of $S_{\alpha}$. Since $T_{\alpha} \in \mathbf{G}_{n}$ for all $\alpha$, it follows that $\left\langle\left\{x_{i}^{\gamma}\right\}\right\rangle$ is a subgroup of $\prod_{\alpha \in A} T_{\alpha}$ and therefore also of $F$.

LEMma 4.2. Let $\mathbf{V}=V\left(\left\{S_{\alpha} \mid \alpha \in A\right\}\right)$ where $S_{\alpha} \in \mathbf{R S}_{n}$. Then $\mathbf{V} \cap \mathbf{G}_{n}$ is generated by the subgroups of the $S_{\alpha}$.

PROOF. Let $\mathbf{W}$ denote the variety generated by the subgroups of the $S_{\alpha}$. Clearly $\mathbf{W} \subseteq$ $\mathbf{V} \cap \mathbf{G}_{n}$. Now let $u\left(x_{1}, \ldots, x_{m}\right)=v\left(x_{1}, \ldots, x_{m}\right)$ be any identity not satisfied by $\mathbf{V} \cap \mathbf{G}_{n}$. Then there must exist $S \in \mathbf{V} \cap \mathbf{G}_{n}$ and a substitution $\varphi: x_{i} \mapsto a_{i}$ of the $x_{i}$ into $S$ such that $u\left(a_{1}, \ldots, a_{m}\right) \neq v\left(a_{1}, \ldots, a_{m}\right)$. Extend $\varphi$ to a homomorphism $\varphi: F=$ $F\left(x_{0}, x_{1}, \ldots, x_{m}\right) \rightarrow S$ by defining $\varphi\left(x_{0}\right)=e$, the identity of $S$. Let $x_{i}^{\gamma}(1 \leq i \leq m)$, $u^{\gamma}$ and $v^{\gamma}$ be defined as above. Then

$$
\begin{aligned}
\varphi\left(x_{i}^{\gamma}\right) & =\varphi\left(\left(x_{0} w x_{0}\right)^{n} x_{0}^{n} x_{i} x_{0}^{n}\right) \\
& =\left(e w\left(a_{1}, \ldots, a_{m}\right) e\right)^{n} e^{n} a_{i} e^{n} \\
& =a_{i}
\end{aligned}
$$


so that

$$
\begin{aligned}
\varphi u^{\gamma}\left(x_{0}, x_{1}, \ldots, x_{m}\right) & =\varphi u\left(x_{1}^{\gamma}, \ldots, x_{m}^{\gamma}\right) \\
& =u\left(a_{1}, \ldots, a_{m}\right) \\
& \neq v\left(a_{1}, \ldots, a_{m}\right) \\
& =\varphi v\left(x_{1}^{\gamma}, \ldots, x_{m}^{\gamma}\right) \\
& =\varphi v^{\gamma}\left(x_{0}, x_{1}, \ldots, x_{m}\right) .
\end{aligned}
$$

Thus $S$ does not satisfy the identity $u^{\gamma}=v^{\gamma}$. Since $S \in \mathbf{V}$ and $\mathbf{V}$ is the variety generated by the $S_{\alpha}$, it follows that there exists $\alpha \in A$ and a homomorphism $\theta: F \rightarrow S_{\alpha}$ such that $\theta u^{\gamma} \neq \theta v^{\gamma}$. Setting $b_{i}=\theta\left(x_{i}^{\gamma}\right)$, we obtain

$$
\begin{aligned}
u\left(b_{1}, \ldots, b_{m}\right) & =\theta u\left(x_{1}^{\gamma}, \ldots, x_{m}^{\gamma}\right) \\
& =\theta u^{\gamma}\left(x_{0}, x_{1}, \ldots, x_{m}\right) \\
& \neq \theta v^{\gamma}\left(x_{0}, x_{1}, \ldots, x_{m}\right) \\
& =\theta v\left(x_{1}^{\gamma}, \ldots, x_{m}^{\gamma}\right) \\
& =v\left(b_{1}, \ldots, b_{m}\right) .
\end{aligned}
$$

By Lemma 4.1, $\left\langle\left\{x_{i}^{\gamma}\right\}\right\rangle$ is a subgroup of $F$. Hence $\theta\left\langle\left\{x_{i}^{\gamma}\right\}\right\rangle=\left\langle\left\{b_{i}\right\}\right\rangle$ must be a subgroup, $T_{\alpha}$ say, of $S_{\alpha}$. Thus $T_{\alpha} \in \mathbf{W}$, and does not satisfy the identity $u=v$. By the arbitrary nature of the identity $u=v$, it follows that $\mathbf{V} \cap \mathbf{G}_{n} \subseteq \mathbf{W}$, and equality prevails.

THEOREM 4.3. The mapping

$$
\chi_{\mathbf{G}_{n}}: \mathbf{V} \mapsto \mathbf{V} \cap \mathbf{G}_{n} \quad\left(\mathbf{V} \in \mathcal{L}\left(\mathbf{R S}_{n}\right)\right)
$$

is a complete retraction of $\mathcal{L}\left(\mathbf{R S}_{n}\right)$ onto $\mathcal{L}\left(\mathbf{G}_{n}\right)$. Consequently $\chi_{\mathbf{G}_{n}}$ induces a complete congruence, and, for any $\mathbf{U} \in \mathcal{L}\left(\mathbf{G}_{n}\right)$, the class of $\mathbf{U}$ in this complete congruence is an interval, which will be denoted by $\left[\mathbf{U}, R S_{n}(\mathbf{U})\right]$.

PROOF. It is clear that $\chi_{\mathbf{G}_{n}}$ respects arbitrary intersections. Now let $\mathbf{V}_{\alpha} \in \mathcal{L}\left(\mathbf{R S}_{n}\right), \alpha \in A$. Trivially,

$$
\left(\bigvee_{\alpha \in A} \mathbf{V}_{\alpha}\right) \cap \mathbf{G}_{n} \supseteq \bigvee_{\alpha \in A}\left(\mathbf{V}_{\alpha} \cap \mathbf{G}_{n}\right) .
$$

So let $G \in\left(\bigvee_{\alpha \in A} \mathbf{V}_{\alpha}\right) \cap \mathbf{G}_{n}$. Then there exist $S_{\beta} \in \bigcup_{\alpha \in A} \mathbf{V}_{\alpha}, \beta \in B$, such that $G \in V\left(\left\{S_{\beta} \mid \beta \in B\right\}\right)$. By Lemma 4.2, $G$ is contained in the variety generated by the subgroups of the $S_{\beta}$. Consequently, $G \in \bigvee_{\beta \in B}\left(\mathbf{V}_{\beta} \cap \mathbf{G}_{n}\right)$, so that

$$
\left(\bigvee_{\alpha \in A} \mathbf{V}_{\alpha}\right) \cap \mathbf{G}_{n} \subseteq \bigvee_{\alpha \in A}\left(\mathbf{V}_{\alpha} \cap \mathbf{G}_{n}\right)
$$

whence equality prevails. 
THEOREM 4.4. Let $\mathbf{U}=\left[u_{\alpha}=v_{\alpha}\right]_{\alpha \in A} \in \mathcal{L}\left(\mathbf{G}_{n}\right)$ where, for each $\alpha \in A$, $u_{\alpha}=u_{\alpha}\left(x_{1}, \ldots, x_{m}\right), v_{\alpha}=v_{\alpha}\left(x_{1}, \ldots, x_{m}\right) \in X^{+}$. Then:

(i) $R S_{n}(\mathbf{U})=\left\{S \in \mathbf{R S}_{n} \mid\right.$ all subgroups of $S$ lie in $\left.\mathbf{U}\right\}$;

(ii) $R S_{n}(\mathbf{U})=\left[\mathrm{I}_{n}, \mathrm{II}_{n}, \mathrm{III}_{n}, u_{\alpha}^{\gamma}=v_{\alpha}^{\gamma}\right]_{\alpha \in A}$.

PROOF. (i) This follows easily from Lemma 4.2.

(ii) See [2, Theorem 3.4 and Corollary 3.5].

\section{The interval $\left[\mathbf{B}_{2}, \mathbf{B}_{2} \vee G_{n}\right]$}

In this section we analyse the interval $\left[\mathbf{B}_{\mathbf{2}}, \mathbf{B}_{\mathbf{2}} \vee \mathbf{G}_{n}\right]$ in more detail.

LeMma 5.1. Let $\mathbf{V} \in\left[\mathbf{B}_{\mathbf{2}},\left[\mathrm{I}_{n}, \mathrm{II}_{n}, \mathrm{III}_{n}, x^{n} y^{n}=y^{n} x^{n}\right]\right], n>1$. Then

$$
\mathbf{V}=\left(\mathbf{V} \cap \mathbf{G}_{n}\right) \vee \mathbf{B}_{\mathbf{2}}
$$

Proof. Clearly $\left(\mathbf{V} \cap \mathbf{G}_{n}\right) \vee \mathbf{B}_{2} \subseteq \mathbf{V}$. By Corollary 2.4, $\mathbf{V}$ is exact. So consider any $S=\mathcal{M}^{0}(I, G, \Lambda ; P) \in \mathbf{V}$. If $G$ is trivial, then

$$
\begin{aligned}
S & \in \mathbf{V} \cap \mathbf{A}_{\mathbf{2}}^{*} \\
& =\mathbf{B}_{\mathbf{2}} \quad(\text { by Lemma 2.3) } \\
& \subseteq\left(\mathbf{V} \cap \mathbf{G}_{n}\right) \vee \mathbf{B}_{\mathbf{2}} .
\end{aligned}
$$

Now assume that $G$ is nontrivial. Since $S$ satisfies the identity $x^{n} y^{n}=y^{n} x^{n}$, we may, by Lemma 3.1, assume that $P$ is a $\{0,1\}$-matrix and that $I$ and $\Lambda$ are partitioned as in Lemma 3.1(ii). Let

$$
K=\prod_{\alpha \in A \cup\{0\}} G_{\alpha},
$$

where $G_{\alpha}=G$ for all $\alpha$. Clearly $K \in V(G) \cap \mathbf{G}_{n}$. Let $g \in G \backslash\{1\}$, and for $i \in I$ define

$$
u_{i}(\alpha)= \begin{cases}g & \text { if } i \in I_{\alpha}, \\ 1 & \text { otherwise }\end{cases}
$$

We identify $G$ with $\{k \in K \mid k(\alpha)=1$ for all $\alpha \neq 0\}$, where $k(\alpha)$ denotes the $\alpha$-component of the element $k$ from the direct product. It is straightforward to verify that the mapping $i \mapsto u_{i}$ satisfies the conditions $\mathrm{K}(\mathrm{i})$ and $\mathrm{K}(\mathrm{ii})$. So we can let $T$ be defined as in the discussion preceding Theorem 3.2. Then

$$
\begin{aligned}
S & \in V(G) \vee V(S / \mathcal{H}) \quad \text { by Theorem 3.2(iv) and (vi) } \\
& \subseteq\left(\mathbf{V} \cap \mathbf{G}_{n}\right) \vee\left(\mathbf{N B}_{2} \cap V(S)\right) \quad \text { by Theorem 3.2(v) } \\
& \subseteq\left(\mathbf{V} \cap \mathbf{G}_{n}\right) \vee \mathbf{B}_{2} \quad \text { by Lemma 1.1(i). }
\end{aligned}
$$

Therefore $\mathbf{V} \subseteq\left(\mathbf{V} \cap \mathbf{G}_{n}\right) \vee \mathbf{B}_{\mathbf{2}}$, and equality prevails. 
Lemma 5.2. Let $\mathbf{U} \in \mathcal{L}\left(\mathbf{G}_{n}\right)(n>1)$ and $\mathbf{V}=R S_{n}(\mathbf{U}) \cap\left[x^{n} y^{n}=y^{n} x^{n}\right]$.

(i) $\mathbf{V}$ is the largest subvariety of $\left[\mathrm{I}_{n}, \mathrm{III}_{n}, \mathrm{III}_{n}, x^{n} y^{n}=y^{n} x^{n}\right]$ such that $\mathbf{V} \cap$ $\mathbf{G}_{n}=\mathbf{U}$.

(ii) $\mathbf{V}=\mathbf{U} \vee \mathbf{B}_{2}$.

Proof. (i) This is clear from the definition of $R S_{n}(\mathbf{U})$ in Theorem 4.3.

(ii) Since $\mathbf{V} \in\left[\mathbf{B}_{2},\left[\mathrm{I}_{n}, \mathrm{II}_{n}, \mathrm{III}_{n}, x^{n} y^{n}=y^{n} x^{n}\right]\right]$, we have

$$
\begin{aligned}
\mathbf{V} & =\left(\mathbf{V} \cap \mathbf{G}_{n}\right) \vee \mathbf{B}_{\mathbf{2}} \quad \text { by Lemma } 5.1 \\
& =\mathbf{U} \vee \mathbf{B}_{\mathbf{2}} \quad \text { by (i). }
\end{aligned}
$$

COROllary 5.3. For $n>1$,

$$
\mathbf{B}_{\mathbf{2}} \vee \mathbf{G}_{n}=\left[\mathrm{I}_{n}, \mathrm{II}_{n}, \mathrm{III}_{n}, x^{n} y^{n}=y^{n} x^{n}\right]
$$

is the largest subvariety of $\mathbf{R S}_{n}$ whose intersection with $\mathbf{R S}_{\mathbf{1}}$ is $\mathbf{B}_{\mathbf{2}}$.

PRoOf. We have

$$
\begin{aligned}
{\left[\mathrm{I}_{n}, \mathrm{II}_{n}, \mathrm{III}_{n}, x^{n} y^{n}=y^{n} x^{n}\right] } & =\mathbf{R S}_{n} \cap\left[x^{n} y^{n}=y^{n} x^{n}\right] \\
& =R S_{n}\left(\mathbf{G}_{n}\right) \cap\left[x^{n} y^{n}=y^{n} x^{n}\right] \\
& =\mathbf{B}_{\mathbf{2}} \vee \mathbf{G}_{n} \quad \text { by Lemma 5.2(ii), }
\end{aligned}
$$

so that the equality holds. In combination with the identity $x^{2}=x^{3}$, the identities $\mathrm{I}_{n}, \mathrm{II}_{n}$ and $\mathrm{III}_{n}$ reduce to the defining identities for $\mathbf{A}_{\mathbf{2}}^{*}\left(=\mathbf{R} \mathbf{S}_{\mathbf{1}}\right)$ in Lemma 1.1(v), so that

$$
\begin{aligned}
& {\left[\mathrm{I}_{n}, \mathrm{II}_{n}, \mathrm{III}_{n}, x^{n} y^{n}=y^{n} x^{n}\right] \cap \mathbf{R S}_{\mathbf{1}}} \\
& \quad=\left[x^{2}=x^{3}, x y x=x y x y x, x y x z x=x z x y x, x^{2} y^{2}=y^{2} x^{2}\right] \\
& \quad=\left[x^{2}=x^{3}, x y x=x y x y x, x^{2} y^{2}=y^{2} x^{2}\right],
\end{aligned}
$$

since the identity $x y x z x=x z x y x$ may be obtained from the other identities. Thus, by Lemma 1.1(i),

$$
\left[\mathrm{I}_{n}, \mathrm{II}_{n}, \mathrm{III}_{n}, x^{n} y^{n}=y^{n} x^{n}\right] \cap \mathbf{R S}_{\mathbf{1}}=\mathbf{B}_{\mathbf{2}} .
$$

By Lemma 2.3, $\left[\mathrm{I}_{n}, \mathrm{II}_{n}, \mathrm{III}_{n}, x^{n} y^{n}=y^{n} x^{n}\right]$ is then the largest subvariety of $\mathbf{R S}_{n}$ whose intersection with $\mathbf{R S}_{\mathbf{1}}$ is $\mathbf{B}_{\mathbf{2}}$.

\section{The interval $\left[\mathbf{B}_{2}, \mathrm{NB}_{2} \vee \mathrm{G}_{n}\right]$}

We are now ready to consider the interval $\left[\mathbf{B}_{\mathbf{2}}, \mathbf{N B}_{\mathbf{2}} \vee \mathbf{G}_{n}\right]$. We begin with a description of the interval $\left[\mathbf{B}_{2}, \mathbf{N B}_{2}\right]$.

LEMma 6.1. The interval $\left[\mathbf{B}_{\mathbf{2}}, \mathbf{N B}_{\mathbf{2}}\right]$ consists of the varieties $\mathbf{B}_{\mathbf{2}}, \mathbf{L} \mathbf{N B}_{\mathbf{2}}, \mathbf{R} \mathbf{N B}_{\mathbf{2}}$ and $\mathrm{NB}_{2}$.

Proof. See [9, Theorem 8.1]. 


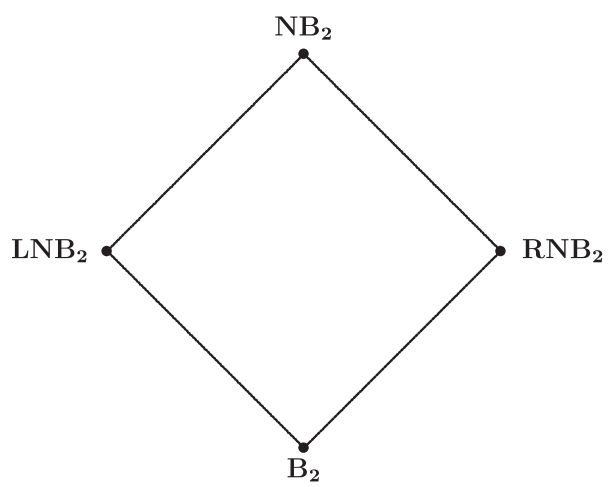

THEOREM 6.2. Let $\mathbf{V} \in\left[\mathbf{B}_{\mathbf{2}},\left[\mathrm{I}_{n}, \mathrm{II}_{n}, \mathrm{III}_{n}, a x^{n} y^{n} b=a y^{n} x^{n} b\right]\right], n>1$. Then

$$
\mathbf{V}=\left(\mathbf{V} \cap \mathbf{G}_{n}\right) \vee\left(\mathbf{V} \cap \mathbf{N B}_{2}\right)
$$

and, in particular, $\mathbf{V}$ is exact.

PROOF. Let $\mathbf{U}$ denote the subvariety of $\mathbf{V}$ generated by the completely 0 -simple semigroups in $\mathbf{V}$. The first step will be to show that $\mathbf{U}=\mathbf{V}$. We will do this by showing that any identity not satisfied by $\mathbf{V}$ is also not satisfied by $\mathbf{U}$.

First note that we must have $\mathbf{V} \cap \mathbf{N B}_{\mathbf{2}} \in\left[\mathbf{B}_{\mathbf{2}}, \mathbf{N B}_{\mathbf{2}}\right]$, so that by Lemma 6.1, $\mathbf{V} \cap \mathbf{N B}_{\mathbf{2}}$ is one of the varieties $\mathbf{B}_{\mathbf{2}}, \mathbf{L N B}_{\mathbf{2}}, \mathbf{R} \mathbf{N B}_{\mathbf{2}}$ or $\mathbf{N B}_{\mathbf{2}}$, and is, therefore, an exact variety. It is also clear that

$$
\mathbf{B}_{\mathbf{2}},\left(\mathbf{V} \cap \mathbf{G}_{n}\right) \vee\left(\mathbf{V} \cap \mathbf{N B}_{2}\right) \subseteq \mathbf{U}
$$

Let $u, v \in X^{+}$and $u=v$ be an identity not satisfied by $\mathbf{V}$. Let $\rho$ be the fully invariant congruence on $X^{+}$corresponding to $\mathbf{V}$, and let $\gamma: X^{+} \rightarrow X^{+} / \rho$ be the natural homomorphism. Then $\gamma(u) \neq \gamma(v)$.

If $\gamma(u)$ and $\gamma(v)$ are both regular elements in $X^{+} / \rho$, then, by Lemma 1.7, there exists a completely 0 -simple semigroup $S$ and a surjective homomorphism $\theta: X^{+} / \rho \rightarrow S$ with $\theta \gamma(u) \neq \theta \gamma(v)$. Since $S$ is a homomorphic image of $X^{+} / \rho$, it follows that $S \in \mathbf{V}$, and therefore $S \in \mathbf{U}$, so that $\mathbf{U}$ does not satisfy the identity $u=v$. Consequently we may assume that one of $\gamma(u), \gamma(v)$ is not regular, say $\gamma(u)$ is not regular.

If $c(u) \neq c(v)$, then $\mathbf{U}$ does not satisfy the identity $u=v$, since $B_{2}$ does not and $B_{2} \in \mathbf{U}$. So we may also assume that $c(u)=c(v)$, and we may argue by induction on $|c(u)|$.

First assume that $|c(u)|=1$, say $c(u)=c(v)=\{x\}$. Then $u=v$ is an identity satisfied by the monogenic free semigroup $\langle x\rangle_{\mathbf{U}}$ in $\mathbf{U}$ on the single generator $\{x\}$. Since $\mathbf{R S}_{n}$ satisfies the identity $x^{2}=x^{n+2}$, it follows that all monogenic semigroups in $\mathbf{R S}_{n}$ have index at most 2. Since $B_{2} \in \mathbf{U},\langle x\rangle_{\mathbf{U}}$ must have index 2. Likewise, the 
monogenic free semigroup $\langle x\rangle_{\mathbf{V}}$ in $\mathbf{V}$ must have index 2. Since $\mathbf{U}$ and $\mathbf{V}$ contain the same groups, it must also be the case that $\langle x\rangle_{\mathbf{U}}$ and $\langle x\rangle_{\mathbf{V}}$ have the same period, so we must have $\langle x\rangle_{\mathbf{U}} \cong\langle x\rangle_{\mathbf{V}}$. (See Howie [3] for a discussion of the structure of monogenic semigroups.) But then $\langle x\rangle_{\mathbf{U}}$ and $\langle x\rangle_{\mathbf{V}}$ will satisfy the same identities and, if $\mathbf{V}$ does not satisfy the identity $u=v$, then neither does $\mathbf{U}$.

So now assume that $|c(u)|=m>1$, and that the claim holds for any identity $u=v$ with $c(u)=c(v)$ and $|c(u)|<m$.

First consider the case where $u$ is a repeated word. By Lemma 1.8, we may assume that $u$ is of the form $u=u_{1} u_{2} \ldots u_{k}$ where the factors $u_{1}, \ldots, u_{k}$ are all covered by cycles. By Lemma 1.6, each of the elements $\gamma\left(u_{i}\right)$ is a regular element of $X^{+} / \rho$. Furthermore, invoking the identities of $\mathbf{R S}_{n}$ and $a x^{n} y^{n} b=a y^{n} x^{n} b$ we obtain

$$
\begin{aligned}
\left(x^{n} y^{n}\right)^{n+1} & =x^{n} y^{n} x^{n} \ldots y^{n} x^{n} y^{n} \\
& =\left(x^{n}\right)^{n+1}\left(y^{n}\right)^{n+1} \\
& =x^{n} y^{n} .
\end{aligned}
$$

Consequently, by Proposition 2.6, the regular elements of $X^{+} / \rho$ form a subsemigroup. Hence

$$
\gamma(u)=\gamma\left(u_{1}\right) \gamma\left(u_{2}\right) \ldots \gamma\left(u_{k}\right)
$$

must also be a regular element in $X^{+} / \rho$. But that would be a contradiction. Hence there must exist a letter $t$ that appears in $u$ exactly once and is not contained in any subword $w$ of $u$ with $h(w)=t(w)$. So we can write

$$
u=u_{1} t u_{2},
$$

where $u_{1}, u_{2} \in X^{*}, t \notin c\left(u_{1} u_{2}\right)$ and $c\left(u_{1}\right) \cap c\left(u_{2}\right)=\emptyset$. Since $c(u)=c(v), t$ must appear in $v$. First suppose that $t$ appears in $v$ at least twice. That means that

$$
v=v_{1} t v_{2} t v_{3},
$$

where $v_{1}, v_{2}, v_{3} \in X^{*}$. Define a homomorphism $\theta: X^{+} \rightarrow B_{2}$ by

$$
\theta(x)= \begin{cases}a & \text { if } x=t \\ a b & \text { if } x \in c\left(u_{1}\right) \\ b a & \text { if } x \in c\left(u_{2}\right) \\ b a & \text { otherwise }\end{cases}
$$

Then $\theta(u)=a \neq 0, \theta(v)=0$ and $\theta(u) \neq \theta(v)$. Thus the identity $u=v$ does not hold in $B_{2}$.

Now suppose that $t$ appears in $v$ exactly once:

$$
v=v_{1} t v_{2}, \quad t \notin c\left(v_{1} v_{2}\right) \quad \text { and } \quad v_{1}, v_{2} \in X^{*}
$$


If either $c\left(u_{1}\right) \cap c\left(v_{2}\right) \neq \emptyset$ or $c\left(u_{2}\right) \cap c\left(v_{1}\right) \neq \emptyset$, then, with $\theta$ as defined above,

$$
\theta(u)=a \neq 0=\theta(v),
$$

and again the identity $u=v$ does not hold in $B_{2}$. So we can assume that $c\left(u_{1}\right)=c\left(v_{1}\right)$ and $c\left(u_{2}\right)=c\left(v_{2}\right)$.

The argument is now broken down into the following cases:

(a) $\quad u=u_{1} t u_{2} \quad$ where $c\left(u_{1}\right) \neq \emptyset \neq c\left(u_{2}\right)$;

(b) $\quad u=t u_{2} \quad$ where $c\left(u_{2}\right) \neq \varnothing$ and the variables of $u_{2}$ are all repeated;

(c) $u=u_{1} t \quad$ where $c\left(u_{1}\right) \neq \varnothing$ and the variables of $u_{1}$ are all repeated;

(d) $\quad u=s u_{3} t \quad$ where the variable $s$ (as well as $t$ ) appears only once, $c\left(u_{3}\right) \neq \emptyset$ and the variables of $u_{3}$ are all repeated.

(Note that in case (d) we may assume that $c\left(u_{3}\right) \neq \emptyset$, since otherwise $u=s t$ and, by the arguments above, we must also have $v=s t$, so that $\mathbf{V}$ would satisfy the identity $s t=s t$ or $u=v$ trivially, which would be a contradiction.)

In case (a), by the above argument, we also have $v=v_{1} t v_{2}$ where $c\left(u_{1}\right)=c\left(v_{1}\right)$ and $c\left(v_{2}\right)=c\left(u_{2}\right)$. Necessarily, at least one of the identities $u_{1} t=v_{1} t, t u_{2}=t v_{2}$ does not hold in $\mathbf{V}$, say $u_{1} t=v_{1} t$ does not hold in $\mathbf{V}$. Then

$$
\left|c\left(u_{1} t\right)\right|=\left|c\left(v_{1} t\right)\right|<m
$$

and we may apply the induction hypothesis to conclude that $\mathbf{U}$ does not satisfy the identity $u_{1} t=v_{1} t$ either.

Hence there exists $S=M^{0}(I, G, \Lambda ; P) \in \mathbf{U}$ and a homomorphism $\theta: X^{+} \rightarrow S$ with $\theta\left(u_{1} t\right) \neq \theta\left(v_{1} t\right)$. At least one of these elements must be nonzero, and therefore $\theta(t) \neq 0$. Let $e$ be any idempotent in the $\mathcal{L}$-class of $\theta(t)$. Now define $\varphi: X^{+} \rightarrow S$ by

$$
\varphi(x)= \begin{cases}\theta(x) & \text { if } x \in c\left(u_{1} t\right)=c\left(v_{1} t\right) \\ e & \text { otherwise }\end{cases}
$$

Then

$$
\begin{aligned}
\varphi(u) & =\varphi\left(u_{1} t\right) \varphi\left(u_{2}\right) \\
& =\theta\left(u_{1} t\right) e \\
& =\theta\left(u_{1} t\right) \\
& \neq \theta\left(v_{1} t\right) \\
& =\theta\left(v_{1} t\right) e \\
& =\varphi\left(v_{1} t v_{2}\right) \\
& =\varphi(v) .
\end{aligned}
$$

Hence the identity $u=v$ does not hold in $S$ or $\mathbf{U}$. 
We now proceed to case (d). The arguments in cases (b) and (c) are similar. In case (d) we know from the preceding discussion that $v$ must also have the form $v=s v_{3} t$ where $c\left(v_{3}\right)=c\left(u_{3}\right)$.

We argue by contradiction. Suppose that $\mathbf{U}$ satisfies the identity $u=v$. Since $\mathbf{V} \cap \mathbf{G}_{n}, \mathbf{B}_{\mathbf{2}} \subseteq \mathbf{U}$, it follows that $\mathbf{V} \cap \mathbf{G}_{n}$ and $\mathbf{B}_{2}$ satisfy the identity $u=v$, that is, the identity $s u_{3} t=s v_{3} t$.

But $\mathbf{V} \cap \mathbf{G}_{n}$ is a variety of groups and $s, t \notin c\left(u_{3}\right)=c\left(v_{3}\right)$. Hence $\mathbf{V} \cap \mathbf{G}_{n}$ must satisfy the identity $u_{3}=v_{3}$. By Lemma $1.3, \mathbf{B}_{2}$ also satisfies the identity $u_{3}=v_{3}$, whence $\left(\mathbf{V} \cap \mathbf{G}_{n}\right) \vee \mathbf{B}_{2}$ does also. Let $\left\{u_{\alpha}=v_{\alpha}\right\}_{\alpha \in A}$ be a basis of identities for $\mathbf{V} \cap \mathbf{G}_{n}$, where $u_{\alpha}, v_{\alpha} \in X^{+}$. Then

$$
\begin{aligned}
& \left(\mathbf{V} \cap \mathbf{G}_{n}\right) \vee \mathbf{B}_{2}=R S_{n}\left(\mathbf{V} \cap \mathbf{G}_{n}\right) \cap\left[x^{n} y^{n}=y^{n} x^{n}\right] \quad \text { by Lemma 5.2, } \\
& \quad=\left[\mathrm{I}_{n}, \mathrm{II}_{n}, \mathrm{III}_{n}, u_{\alpha}^{\gamma}=v_{\alpha}^{\gamma}(\alpha \in A), x^{n} y^{n}=y^{n} x^{n}\right] \quad \text { by Theorem 4.4(ii). }
\end{aligned}
$$

Therefore, there exists a deduction

$$
\left\{\mathrm{I}_{n}, \mathrm{II}_{n}, \mathrm{III}_{n}, u_{\alpha}^{\gamma}=v_{\alpha}^{\gamma}(\alpha \in A), x^{n} y^{n}=y^{n} x^{n}\right\} \vdash u_{3}=v_{3},
$$

so that there will also be a deduction

$$
\left\{\mathrm{I}_{n}, \mathrm{II}_{n}, \mathrm{III}_{n}, u_{\alpha}^{\gamma}=v_{\alpha}^{\gamma}(\alpha \in A), a x^{n} y^{n} b=a y^{n} x^{n} b\right\} \vdash u=v .
$$

But

$$
\begin{array}{r}
\left\{\mathrm{I}_{n}, \mathrm{II}_{n}, \mathrm{III}_{n}, u_{\alpha}^{\gamma}=v_{\alpha}^{\gamma}(\alpha \in A), a x^{n} y^{n} b=a y^{n} x^{n} b\right\} \\
=R S_{n}\left(\mathbf{V} \cap \mathbf{G}_{n}\right) \cap\left[a x^{n} y^{n} b=a y^{n} x^{n} b\right] \supseteq \mathbf{V},
\end{array}
$$

which implies that $\mathbf{V}$ must satisfy the identity $u=v$, a contradiction. Therefore the identity $u=v$ must fail in $\mathbf{U}$.

We have now shown that the identity $u=v$ fails in $\mathbf{U}$ in all cases. Thus $\mathbf{V}=\mathbf{U}$, and $\mathbf{V}$ is generated by its completely 0 -simple members.

We are now able to establish the desired decomposition of $\mathbf{V}$. Let

$$
S=\mathcal{M}^{0}(I, G, \Lambda ; P) \in \mathbf{V} .
$$

If $G$ is trivial, then

$$
\begin{aligned}
S & \in \mathbf{V} \cap \mathbf{A}_{\mathbf{2}}^{*} \\
& \left.\subseteq \mathbf{V} \cap \mathbf{N B}_{\mathbf{2}} \quad \text { (by Lemma 1.1(iv) }\right) \\
& \subseteq\left(\mathbf{V} \cap \mathbf{G}_{n}\right) \vee\left(\mathbf{V} \cap \mathbf{N B}_{\mathbf{2}}\right) .
\end{aligned}
$$

Now assume that $G$ is nontrivial. In this case, $S$ satisfies the hypothesis of Lemma 3.1, and, as in Lemma 5.1, we may construct the semigroup $T$ according to the discussion preceding Theorem 3.2. Then

$S \in V(T)$ by Theorem 3.2(iv), $T \in V(K) \vee V(S / \mathcal{H}) \quad$ by Theorem 3.2(v), (vi), 
where, by the choice of $K$,

$$
V(K) \subseteq V(G) \subseteq \mathbf{V} \cap \mathbf{G}_{n},
$$

while

$$
\begin{aligned}
V(S / \mathcal{H}) \in \mathbf{V} \cap \mathbf{R S}_{\mathbf{1}} & \subseteq \mathbf{V} \cap\left[a x^{n} y^{n} b=a y^{n} x^{n} b\right] \cap \mathbf{R S}_{\mathbf{1}} \\
& =\mathbf{V} \cap \mathbf{N B}_{\mathbf{2}} .
\end{aligned}
$$

Thus

$$
S \in V(T) \subseteq\left(\mathbf{V} \cap \mathbf{G}_{n}\right) \vee\left(\mathbf{V} \cap \mathbf{N B}_{2}\right),
$$

so that

$$
\mathbf{V} \subseteq\left(\mathbf{V} \cap \mathbf{G}_{n}\right) \vee\left(\mathbf{V} \cap \mathbf{N B}_{\mathbf{2}}\right),
$$

whence equality prevails, since the reverse inclusion is trivial.

There are some interesting special cases of Theorem 6.2.

COROLlary 6.3. The following hold:

(i) $\mathbf{L N B}_{2} \vee \mathbf{G}_{n}=\left[\mathrm{I}_{n}, \mathrm{II}_{n}, \mathrm{III}_{n}, a x^{n} y^{n}=a y^{n} x^{n}\right]$;

(ii) $\mathbf{R N B}_{2} \vee \mathbf{G}_{n}=\left[\mathrm{I}_{n}, \mathrm{II}_{n}, \mathrm{III}_{n}, x^{n} y^{n} b=y^{n} x^{n} b\right]$;

(iii) $\mathbf{N B}_{\mathbf{2}} \vee \mathbf{G}_{n}=\left[\mathrm{I}_{n}, \mathrm{II}_{n}, \mathrm{III}_{n}, a x^{n} y^{n} b=a y^{n} x^{n} b\right]$.

ProOF. (i) Let $\mathbf{V}$ denote the variety on the right-hand side of the equation. Clearly $\mathbf{V} \cap \mathbf{G}_{n}=\mathbf{G}_{n}$, while, by Lemma 1.1, $\mathbf{V} \cap \mathbf{N B}_{\mathbf{2}}=\mathbf{L} \mathbf{N B}_{\mathbf{2}}$. Hence, by Theorem 6.2,

$$
\begin{aligned}
\mathbf{V} & =\left(\mathbf{V} \cap \mathbf{G}_{n}\right) \vee\left(\mathbf{V} \cap \mathbf{N B}_{\mathbf{2}}\right) \\
& =\mathbf{G}_{n} \vee \mathbf{L N B}_{\mathbf{2}} .
\end{aligned}
$$

The proofs of (ii) and (iii) follow similarly.

\section{Exact varieties do not form a sublattice}

It is evident that the join of exact varieties is again an exact variety. However, as we will now show, the intersection of two exact varieties need not be exact, so that the exact varieties do not form a sublattice of $\mathcal{L}\left(\mathbf{R S}_{n}\right)$.

EXAMPLE 7.1. Let $p$ and $q$ be distinct primes dividing $n$, and $\langle g\rangle,\langle h\rangle$ be (multiplicative) cyclic groups of orders $p$ and $q$, respectively, with generators $g$ and $h$. Let $\mathbf{3}=\{1,2,3\}$ and

$$
S_{1}=\mathcal{M}^{0}\left(\mathbf{3},\langle g\rangle, \mathbf{3} ; P_{1}\right), \quad S_{2}=\mathcal{M}^{0}\left(\mathbf{3},\langle h\rangle, \mathbf{3} ; P_{2}\right),
$$

where

$$
P_{1}=\left[\begin{array}{lll}
1 & 1 & 0 \\
1 & g & 0 \\
0 & 0 & 1
\end{array}\right] \text { and } P_{2}=\left[\begin{array}{lll}
1 & 1 & 0 \\
1 & h & 0 \\
0 & 0 & 1
\end{array}\right]
$$


Then

$$
\mathbf{V}_{\mathbf{1}}=V\left(S_{1}\right), \quad \mathbf{V}_{\mathbf{2}}=V\left(S_{2}\right)
$$

are both exact varieties. Also, $\mathbf{V}_{\mathbf{1}}$ satisfies $x^{p+2}=x^{2}$ and $\mathbf{V}_{\mathbf{2}}$ satisfies $x^{q+2}=x^{2}$, so that $\mathbf{V}_{\mathbf{1}} \cap \mathbf{V}_{\mathbf{2}}$ satisfies $x^{3}=x^{2}$.

Thus $\mathbf{V}_{\mathbf{1}} \cap \mathbf{V}_{\mathbf{2}}$ is an aperiodic variety. But both $\mathbf{V}_{\mathbf{1}}$ and $\mathbf{V}_{\mathbf{2}}$ satisfy the identity $\left(a x^{n} y^{n} b\right)^{n}=\left(a y^{n} x^{n} b\right)^{n}$, so that any aperiodic exact variety contained in $\mathbf{V}_{\mathbf{1}} \cap \mathbf{V}_{\mathbf{2}}$ must satisfy the identity $\left(a x^{2} y^{2} b\right)^{2}=\left(a y^{2} x^{2} b\right)^{2}$ as well as the identity $x^{2}=x^{3}$. However, any aperiodic completely 0-simple semigroup $\mathcal{M}^{0}(I, G, \Lambda ; P)$ satisfying these identities must also satisfy the identity $a x^{2} y^{2} b=a y^{2} x^{2} b$, and so lie in $\mathbf{N B}_{\mathbf{2}}$. Clearly $\mathbf{N B}_{\mathbf{2}} \subseteq \mathbf{V}_{\mathbf{1}} \cap \mathbf{V}_{\mathbf{2}}$, so that $\mathbf{N B}_{\mathbf{2}}$ is the largest exact variety contained in $\mathbf{V}_{\mathbf{1}} \cap \mathbf{V}_{\mathbf{2}}$. In addition, $\mathbf{V}_{\mathbf{1}} \cap \mathbf{V}_{\mathbf{2}}$ is properly contained in $\mathbf{A}_{\mathbf{2}}^{*}$.

In $S_{1}$, let

$$
e=(1,1,1), \quad f=(1,1,2), \quad a=(1,1,3), \quad b=(1, g, 3), \quad c=(2,1,3) .
$$

Then $T_{1}=\{e, f, a, b, c, 0\}$ is a subsemigroup of $S_{1}$ with multiplication table:

\begin{tabular}{|c|c|c|c|c|c|}
\hline & $e$ & $f$ & $a$ & $b$ & $c$ \\
\hline$e$ & $e$ & $f$ & $a$ & $b$ & $a$ \\
\hline$f$ & $e$ & $f$ & $a$ & $b$ & $b$ \\
\hline$a$ & 0 & 0 & 0 & 0 & 0 \\
\hline$b$ & 0 & 0 & 0 & 0 & 0 \\
\hline$c$ & 0 & 0 & 0 & 0 & 0 \\
\hline
\end{tabular}

Furthermore, with the substitution

$$
a \mapsto e, \quad x \mapsto e, \quad y \mapsto f, \quad b \mapsto c
$$

we find that

$$
\begin{aligned}
& a x^{2} y^{2} b \longmapsto e e^{2} f^{2} c=b, \\
& a y^{2} x^{2} b \longmapsto e f^{2} e^{2} c=a,
\end{aligned}
$$

so that $T_{1}$ does not satisfy the identity $a x^{2} y^{2} b=a y^{2} x^{2} b$. Consequently, $T_{1} \notin \mathbf{N B}_{2}$. However, $T_{1}$ is aperiodic, whence $\mathbf{N B}_{\mathbf{2}} \vee V\left(T_{1}\right)$ is aperiodic but not equal to $\mathbf{N B}_{2}$. On the other hand, $T_{1} \in V\left(S_{1}\right)$, which satisfies the identity $\left(a x^{n} y^{n} b\right)^{n}=\left(a y^{n} x^{n} b\right)^{n}$, and therefore does not contain $A_{2}$. Hence $A_{2} \notin \mathbf{N B}_{2} \vee V\left(T_{1}\right)$. Consequently,

$$
\mathbf{N B}_{2} \vee V\left(T_{1}\right) \in\left[\mathbf{N B}_{2}, \mathbf{A}_{2}^{*}\right],
$$

but is not exact.

In $S_{2}$, let

$$
e=(1,1,1), \quad f=(1,1,2), \quad a=(1,1,3), \quad b=(1, h, 3), \quad c=(2,1,3) .
$$


Then $T_{2}=\{e, f, a, b, c, 0\}$ is a subsemigroup of $S_{2}$ with the same multiplication table as $T_{1}$. Thus $T_{1} \cong T_{2}$. Therefore

$$
\mathbf{N B}_{2} \underset{\neq}{\subset} \mathbf{N B}_{2} \vee V\left(T_{1}\right) \subseteq \mathbf{V}_{1} \cap \mathbf{V}_{\mathbf{2}} \underset{\neq}{\subset} \mathbf{A}_{2}^{*}
$$

so that $\mathbf{V}_{\mathbf{1}} \cap \mathbf{V}_{\mathbf{2}}$ is not an exact variety. Thus the exact varieties do not constitute a sublattice of $\mathcal{L}\left(\mathbf{R S}_{n}\right)$.

A further consequence of Example 7.1 is that the interval $\left[\mathbf{B}_{\mathbf{2}}, \mathbf{N B}_{\mathbf{2}} \vee \mathbf{G}_{n}\right]$ cannot be extended at the upper end without including some nonexact varieties. To see this, let $S=\mathcal{M}^{0}(I, G, \Lambda ; P) \notin \mathbf{N B}_{2} \vee \mathbf{G}_{n}$. If $A_{2} \in V(S)$, then $V(S)$ must also contain the nonexact variety $\mathbf{N B}_{2} \vee V(T)$, where $T$ is as constructed in the example.

That means that $P$ cannot contain any submatrix of the form

$$
\left[\begin{array}{cc}
p_{\lambda i} & p_{\lambda j} \\
p_{\mu i} & 0
\end{array}\right]
$$

where $p_{\lambda i}, p_{\lambda j}$ and $p_{\mu i}$ are nonzero. Otherwise, if $T$ is the corresponding subsemigroup of $S$ then we will have $A_{2} \cong T / \mathcal{H} \in V(S)$, a contradiction. Now consider $P$ as a normalized matrix. If $P$ is a $\{0,1\}$-matrix, then $S$ will satisfy the identity $a x^{n} y^{n} b=a y^{n} x^{n} b$, and $S$ will belong to $\mathbf{N B}_{\mathbf{2}} \vee \mathbf{G}_{n}$, a contradiction again. Hence, there must exist an entry $p_{\lambda i}$ in $P$, when normalized, with $p_{\lambda i} \neq 0,1$. Let $g=p_{\lambda i}$. Then $P$ has a submatrix of the form

$$
\left[\begin{array}{ll}
1 & 1 \\
1 & g
\end{array}\right]
$$

where $g \neq 0,1$. Let $S_{1}=\mathcal{M}^{0}\left(I_{1},\langle g\rangle, \Lambda_{1} ; P_{1}\right)$ denote the corresponding subsemigroup. Now let $S_{2}=\left(S_{1} \times B_{2}\right) / J$, the Rees quotient of $S_{1} \times B_{2}$ modulo the ideal $J=\{(x, y) \mid x=0$ or $y=0\}$. Then $S_{2}$ is a completely 0 -simple semigroup, and we can recognize a subsemigroup of the form

$$
S_{3}=\mathcal{M}^{0}\left(I^{\prime},\langle g\rangle \times\langle 1\rangle, \Lambda^{\prime} ; P_{3}\right),
$$

where

$$
P_{3}=\left[\begin{array}{ccc}
(1,1) & (1,1) & 0 \\
(1,1) & (g, 1) & 0 \\
0 & 0 & (1,1)
\end{array}\right] .
$$

Then $S_{3}$ is of the form of the $S_{i}$ in Example 7.1, and so contains a subsemigroup (isomorphic to) $T$. Therefore

$$
\mathbf{N B}_{\mathbf{2}} \underset{\neq}{\subseteq} \mathbf{N B}_{\mathbf{2}} \vee V(T) \subseteq \mathbf{N B}_{\mathbf{2}} \vee \mathbf{G}_{n} \vee V(S),
$$

where, by Example 7.1, $\mathbf{N B}_{\mathbf{2}} \vee V(T)$ is not exact. 


\section{Further intervals of exact varieties}

The observations of the previous section might lead us to suspect that the situation with regard to exact varieties that do not contain $A_{2}$ and are not contained in $\mathbf{N B}_{\mathbf{2}} \vee \mathbf{G}_{n}$ is somewhat chaotic. As we will now show, that is far from being the case.

THEOREM 8.1. Let $\mathbf{V} \in \mathcal{L}\left(\mathbf{R S}_{n}\right)$ be such that:

(i) $B_{2} \in \mathbf{V}$;

(ii) $\mathbf{V} \subseteq\left[x^{n} y^{n}=\left(x^{n} y^{n}\right)^{n+1}\right] \cap \mathbf{R S}_{n}$;

(iii) $\mathbf{V}$ contains a Rees-matrix semigroup of the form

$$
M_{p}=\mathcal{M}\left(\{1,2\},\langle g\rangle,\{1,2\} ; P_{g}\right),
$$

where $p$ is a prime dividing $n, g$ is a generator of a cyclic group of order $p$, and

$$
P_{g}=\left[\begin{array}{ll}
1 & 1 \\
1 & g
\end{array}\right]
$$

Then $\mathbf{V}$ is an exact variety.

PROOF. Let $\mathbf{U}$ denote the subvariety of $\mathbf{V}$ generated by the completely 0-simple semigroups in $\mathbf{V}$. We will show that any identity not satisfied by $\mathbf{V}$ is also not satisfied by $\mathbf{U}$.

Let $u, v \in X^{+}$and $u=v$ be an identity not satisfied by $\mathbf{V}$. Let $\rho$ be the fully invariant congruence on $X^{+}$corresponding to $\mathbf{V}$, and let $\gamma: X^{+} \rightarrow X^{+} / \rho$ be the natural homomorphism.

The proof now proceeds exactly as in Theorem 6.2 until we reach the point where the argument is broken down into four cases (a), (b), (c) and (d). The argument in case (a) is again identical to that in Theorem 6.2. The cases (b) and (c) are one-sided versions of case (d), and so case (d) remains to be dealt with. Here the argument differs from that of Theorem 6.2.

So let us assume that $\mathbf{V}$ does not satisfy the identity $u=v$, where $u$ and $v$ are of the form

$$
u=s u_{1} t, \quad v=s v_{1} t
$$

and

$$
s, t \in X, \quad u_{1}, v_{1} \in X^{+}, \quad s, t \notin c\left(u_{1}\right)=c\left(v_{1}\right) \neq \emptyset .
$$

Then one of the identities $s u_{1}=s v_{1}$ and $u_{1} t=v_{1} t$ must fail in $\mathbf{V}$, say $s u_{1}=s v_{1}$. Since $\left|c\left(s u_{1}\right)\right|<|c(u)|$, we may invoke the induction hypothesis to conclude that $\mathbf{U}$ does not satisfy the identity $s u_{1}=s v_{1}$ either. Hence there exist $S=\mathcal{M}^{0}(I, G, \Lambda ; P) \in \mathbf{U}$ and a homomorphism $\gamma: X^{+} / \rho \rightarrow S$ such that $\gamma\left(s u_{1}\right) \neq \gamma\left(s v_{1}\right)$. There are several cases to consider.

Case 1: one of $\gamma\left(s u_{1}\right), \gamma\left(s v_{1}\right)$ is nonzero and one is zero. Let us assume that

$$
\gamma\left(s u_{1}\right)=(i, a, \lambda) \neq 0, \quad \gamma\left(s v_{1}\right)=0 .
$$


Since $S$ is regular, there exists $j \in I$ with $p_{\lambda j} \neq 0$. We define $\gamma^{*}: X^{+} / \rho \rightarrow S$ by

$$
\gamma^{*}(x)= \begin{cases}(j, 1, \lambda) & \text { if } x=t \\ \gamma(x) & \text { otherwise }\end{cases}
$$

Then

$$
\begin{aligned}
\gamma^{*}\left(s u_{1} t\right) & =\gamma^{*}\left(s u_{1}\right) \gamma^{*}(t)=\gamma\left(s u_{1}\right)(j, 1, \lambda) \\
& =(i, a, \lambda)(j, 1, \lambda)=\left(i, a p_{\lambda j}, \lambda\right),
\end{aligned}
$$

while

$$
\begin{aligned}
\gamma^{*}\left(s v_{1} t\right) & =\gamma^{*}\left(s v_{1}\right) \gamma^{*}(t)=\gamma\left(s v_{1}\right) \gamma^{*}(t) \\
& =0 \cdot \gamma^{*}(t)=0
\end{aligned}
$$

Thus $\gamma^{*}\left(s u_{1} t\right) \neq \gamma^{*}\left(s v_{1} t\right)$, so that $S$, and therefore also $\mathbf{U}$, does not satisfy the identity $s u_{1} t=s v_{1} t$.

Case 2: both $\gamma\left(s u_{1}\right)$ and $\gamma\left(s v_{1}\right)$ are nonzero, say

$$
\gamma\left(s u_{1}\right)=(i, a, \lambda), \quad \gamma\left(s v_{1}\right)=(j, b, \mu) .
$$

We divide this case into three subcases.

Case 2a: $i \neq j$. Let $k \in I$ be such that $p_{\lambda k} \neq 0$. Then define $\gamma^{*}: X^{+} / \rho \rightarrow S$ by

$$
\gamma^{*}(x)= \begin{cases}(k, 1, \lambda) & \text { if } x=t \\ \gamma(x) & \text { otherwise }\end{cases}
$$

Then

$$
\begin{aligned}
\gamma^{*}\left(s u_{1} t\right) & =\gamma^{*}\left(s u_{1}\right) \gamma^{*}(t)=\gamma\left(s u_{1}\right)(k, 1, \lambda) \\
& =(i, a, \lambda)(k, 1, \lambda)=\left(i, a p_{\lambda k}, \lambda\right),
\end{aligned}
$$

while

$$
\begin{aligned}
\gamma^{*}\left(s v_{1} t\right) & =\gamma^{*}\left(s v_{1}\right) \gamma^{*}(t)=\gamma\left(s v_{1}\right)(k, 1, \lambda) \\
& =(j, b, \mu)(k, 1, \lambda) \\
& = \begin{cases}\left(j, b p_{\mu k}, \lambda\right) & \text { if } p_{\mu k} \neq 0 \\
0 & \text { if } p_{\mu k}=0 \\
& \neq \gamma^{*}\left(s u_{1} t\right) .\end{cases}
\end{aligned}
$$

Thus $S$ and $\mathbf{U}$ do not satisfy the identity $s u_{1} t=s v_{1} t$.

Case $2 b: \lambda \neq \mu$. Let

$$
T=\left(S \times M_{p}\right) / J \quad \text { where } J=\{0\} \times M_{p} .
$$


Since $S, M_{p} \in \mathbf{U}$, we also have $T \in \mathbf{U}$. Let $m \in I$ be such that $p_{\lambda m} \neq 0$. Define $\gamma^{*}: X^{+} / \rho \rightarrow T$ by the following. For $x \in X$,

$$
\gamma^{*}(x)= \begin{cases}(\gamma(x),(1,1,2)) & \text { if } \gamma(x)=(*, *, \lambda) \text { and } x \neq t, \\ (\gamma(x),(1,1,1)) & \text { if } \gamma(x)=(*, *, v), v \neq \lambda, x \neq t, \\ ((m, 1, \lambda),(2,1,2)) & \text { if } x=t\end{cases}
$$

Then

$$
\begin{aligned}
\gamma^{*}\left(s u_{1} t\right) & =\gamma^{*}\left(s u_{1}\right) \gamma^{*}(t) \\
& =\left(\gamma\left(s u_{1}\right), *\right) \gamma^{*}(t) \\
& =((i, a, \lambda),(1,1,2))((m, 1, \lambda),(2,1,2)) \\
& =\left(\left(i, a p_{\lambda m}, \lambda\right),(1, g, 2)\right)
\end{aligned}
$$

while

$$
\begin{aligned}
& \gamma^{*}\left(s v_{1} t\right)=\gamma^{*}\left(s v_{1}\right) \gamma^{*}(t) \\
& =\left(\gamma\left(s v_{1}\right), *\right) \gamma^{*}(t) \\
& =((j, b, \mu),(1,1,1))((m, 1, \lambda),(2,1,2)) \\
& = \begin{cases}\left(\left(j, b p_{\mu m}, \lambda\right),(1,1,2)\right) & \text { if } p_{\mu m} \neq 0 \\
0 & \text { if } p_{\mu m}=0\end{cases} \\
& \neq \gamma^{*}\left(s u_{1} t\right) \text {. }
\end{aligned}
$$

Therefore $T$ and $\mathbf{U}$ do not satisfy the identity $s u_{1} t=s v_{1} t$.

Case 2c: $i=j, \lambda=\mu, a \neq b$.

Let $m \in I$ be such that $p_{\lambda m} \neq 0$. Define $\gamma^{*}: X^{+} / \rho \rightarrow S$ as follows. For $x \in X$,

$$
\gamma^{*}(x)= \begin{cases}(m, 1, \lambda) & \text { if } x=t \\ \gamma(x) & \text { otherwise. }\end{cases}
$$

Then

$$
\begin{aligned}
\gamma^{*}\left(s u_{1} t\right) & =\gamma^{*}\left(s u_{1}\right) \gamma^{*}(t) \\
& =\gamma\left(s u_{1}\right) \gamma^{*}(t) \\
& =(i, a, \lambda)(m, 1, \lambda) \\
& =\left(i, a p_{\lambda m}, \lambda\right)
\end{aligned}
$$

while

$$
\begin{aligned}
\gamma^{*}\left(s v_{1} t\right) & =\gamma^{*}\left(s v_{1}\right) \gamma^{*}(t) \\
& =\gamma\left(s v_{1}\right) \gamma^{*}(t) \\
& =(j, b, \mu)(m, 1, \lambda) \\
& =(i, b, \lambda)(m, 1, \lambda) \\
& =\left(i, b p_{\lambda m}, \lambda\right) \\
& \neq \gamma^{*}\left(s u_{1} t\right) .
\end{aligned}
$$


Thus $S$ and $\mathbf{U}$ do not satisfy the identity $s u_{1} t=s v_{1} t$. In all cases, we have now shown that $\mathbf{U}$ does not satisfy the identity $u=v$. Consequently, $\mathbf{V}=\mathbf{U}$, an exact variety.

The semigroups $M_{p}$ introduced in Theorem 8.1 are important in the study of completely regular semigroups. By Petrich-Reilly [8, Corollary III.5.5], if $S$ is a completely regular semigroup (that is, a union of groups) then the idempotents form a subsemigroup of $S$ if and only if $S$ does not contain any $M_{p}$ as a subsemigroup. Let

$$
\mathbf{M}_{p}=V\left(M_{p}\right) \text {. }
$$

Note that $\mathbf{M}_{p}$ is an exact variety, so that $\mathbf{B}_{2} \vee \mathbf{M}_{p}$ is also. Since $L_{2}$ and $R_{2}$ are subsemigroups of $M_{p}$ we have $\mathbf{L Z}, \mathbf{R Z} \subseteq \mathbf{M}_{p}$.

For each integer $n \geq 1$, let $\mathbf{A}_{n}$ denote the variety of abelian groups of exponent dividing $n$.

Since $\langle g\rangle \in \mathbf{M}_{p}$ it is clear that $\mathbf{A}_{p} \subseteq \mathbf{M}_{p}$ so that

$$
\begin{aligned}
\mathbf{N B}_{\mathbf{2}} \vee \mathbf{A}_{p} & =\mathbf{B}_{\mathbf{2}} \vee \mathbf{L Z} \vee \mathbf{R Z} \vee \mathbf{A}_{p} \\
& \subseteq \mathbf{B}_{\mathbf{2}} \vee \mathbf{M}_{p} .
\end{aligned}
$$

It is straightforward to verify that $M_{p}$ and $B_{2}$ both satisfy the identities

$$
x^{p}=x^{2 p}, \quad x^{p} y x^{p} z x^{p}=x^{p} z x^{p} y x^{p},
$$

so that

$$
\begin{aligned}
& \left(\left(\mathbf{N B}_{\mathbf{2}} \vee \mathbf{G}_{n}\right) \cap\left(\mathbf{B}_{\mathbf{2}} \vee \mathbf{M}_{p}\right)\right) \cap \mathbf{G}_{n} \\
& \quad \subseteq\left[x^{p}=x^{2 p}, x^{p} y x^{p} z x^{p}=x^{p} z x^{p} y x^{p}\right] \cap \mathbf{G}_{n} \\
& \quad=\mathbf{A}_{p} .
\end{aligned}
$$

The reverse inclusion is clear, so that

$$
\left(\left(\mathbf{N B}_{\mathbf{2}} \vee \mathbf{G}_{n}\right) \cap\left(\mathbf{B}_{\mathbf{2}} \vee \mathbf{M}_{p}\right)\right) \cap \mathbf{G}_{n}=\mathbf{A}_{p},
$$

and

$$
\left(\left(\mathbf{N B}_{\mathbf{2}} \vee \mathbf{G}_{n}\right) \cap\left(\mathbf{B}_{\mathbf{2}} \vee \mathbf{M}_{p}\right)\right) \cap \mathbf{N B}_{\mathbf{2}}=\mathbf{N B}_{\mathbf{2}} .
$$

By Theorem 6.2, we thus obtain

$$
\left(\mathbf{N B}_{\mathbf{2}} \vee \mathbf{G}_{n}\right) \cap\left(\mathbf{B}_{\mathbf{2}} \vee \mathbf{M}_{p}\right)=\mathbf{N B}_{\mathbf{2}} \vee \mathbf{A}_{p} .
$$

We can now summarize our discussion.

THEOREM 8.2. The following hold:

(i) Let $\mathbf{V}$ be an exact variety in the interval $\left[\mathbf{B}_{\mathbf{2}},\left[x^{n} y^{n}=\left(x^{n} y^{n}\right)^{n+1}\right]\right.$ $\left.\cap \mathbf{R S}_{n}\right]$ that is not contained in $\mathbf{N B}_{\mathbf{2}} \vee \mathbf{G}_{n}$. Then $\mathbf{V}$ contains a variety of the form $\mathbf{B}_{\mathbf{2}} \vee \mathbf{M}_{p}$ for some prime $p$.

(ii) The only exact varieties in the interval $\left[\mathbf{B}_{\mathbf{2}} \vee \mathbf{A}_{p}, \mathbf{B}_{\mathbf{2}} \vee \mathbf{M}_{p}\right]$ are $\mathbf{B}_{\mathbf{2}} \vee \mathbf{A}_{p}$ and $\mathbf{B}_{\mathbf{2}} \vee \mathbf{M}_{p}$. 
PROOF. (i) Since $\mathbf{V}$ is generated by completely 0 -simple semigroups, there must be a completely 0 -simple semigroup

$$
S=\mathcal{M}^{0}(I, G, \Lambda ; P) \in \mathbf{V} \subseteq\left[x^{n} y^{n}=\left(x^{n} y^{n}\right)^{n+1}\right] \cap \mathbf{R S}_{n}
$$

such that

$$
S \notin \mathbf{N B}_{2} \vee \mathbf{G}_{n} .
$$

Since $A_{2}$ does not satisfy the identity $x^{n} y^{n}=\left(x^{n} y^{n}\right)^{n+1}$, it follows that $A_{2}$ cannot divide $S$. Hence, as in the discussion in Section 7, $S$ must contain a subsemigroup of the form

$$
\begin{gathered}
S_{1}=\mathcal{M}^{0}(\{1,2\},\langle g\rangle,\{1,2\} ; P), \\
P=\left[\begin{array}{ll}
1 & 1 \\
1 & g
\end{array}\right],
\end{gathered}
$$

and $g \neq 1$. Let the order of $g$ be $m$, let $p$ be a prime dividing $m$ and let $M=\left\langle g^{m / p}\right\rangle$. Then $M$ determines a congruence $\rho_{M}$ on $S_{1}$ defined by

$$
\begin{aligned}
a \rho_{M} b \Longleftrightarrow \text { either } & a=(i, h, \lambda), b=(i, k, \lambda) \\
& \text { where } M h=M k \\
\text { or } \quad & a=0=b .
\end{aligned}
$$

(See Howie [3] for a discussion of congruences on completely 0-simple semigroups.) It is easy to see that the quotient $S_{1} / \rho_{M}$ is isomorphic to $M_{p}$, so that $M_{p} \in \mathbf{V}$, and the claim holds.

(ii) By the discussion preceding this theorem, there are no exact varieties in the interval $\left[\mathbf{N B}_{\mathbf{2}} \vee \mathbf{A}_{p}, \mathbf{B}_{\mathbf{2}} \vee \mathbf{M}_{p}\right]$ that are contained in $\mathbf{N B}_{\mathbf{2}} \vee \mathbf{G}_{n}$ other than $\mathbf{N B}_{\mathbf{2}} \vee \mathbf{A}_{p}$. On the other hand, $\mathbf{B}_{\mathbf{2}} \vee \mathbf{M}_{p} \subseteq\left[x^{n} y^{n}=\left(x^{n} y^{n}\right)^{n+1}\right]$, so that by part (i), there are no exact varieties in the interval that are not contained in $\mathbf{N B}_{\mathbf{2}} \vee \mathbf{G}_{n}$ other than $\mathbf{B}_{\mathbf{2}} \vee \mathbf{M}_{p}$, so the claim holds.

Note that the interval $\left[\mathbf{N B}_{\mathbf{2}} \vee \mathbf{A}_{p}, \mathbf{B}_{\mathbf{2}} \vee \mathbf{M}_{p}\right.$ ] contains more than just the endpoints, so that $\mathbf{B}_{\mathbf{2}} \vee \mathbf{M}_{p}$ does not cover $\mathbf{N B}_{\mathbf{2}} \vee \mathbf{A}_{p}$. For instance, with $T_{1}$ as in Section 7, $\mathbf{B}_{2} \vee \mathbf{A}_{2} \vee V\left(T_{1}\right)$ belongs to the interval $\left[\mathbf{B}_{2} \vee \mathbf{A}_{\mathbf{2}}, \mathbf{B}_{\mathbf{2}} \vee \mathbf{M}_{p}\right]$ and differs from both endpoints (but is not exact).

\section{Conclusion}

We can now achieve Kublanovsky's goal with a characterization of the ReesSushkevich varieties that are exact. 


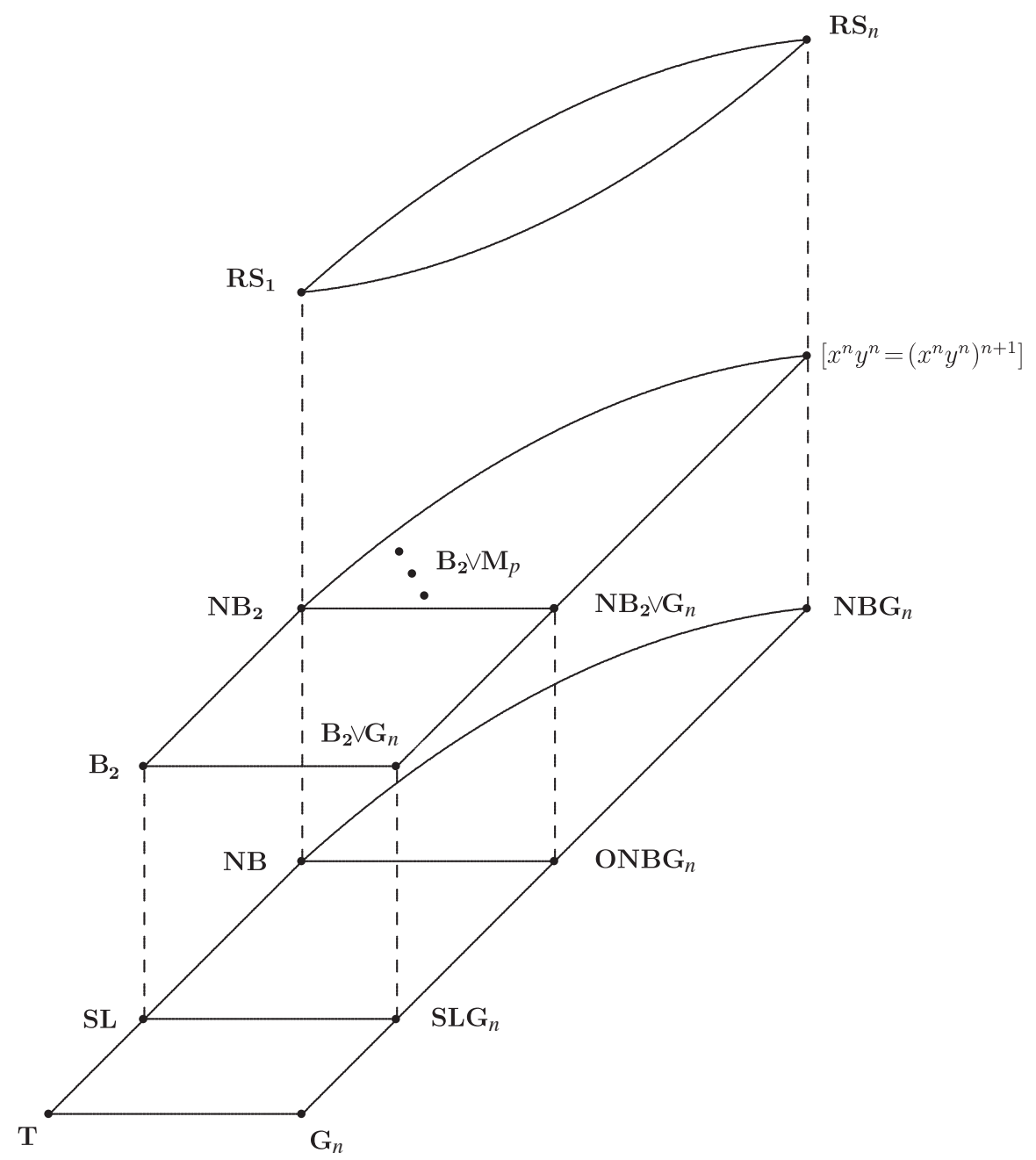

FIGURE 1. The 'lattice' of exact Rees-Sushkevich varieties in $\mathcal{L}\left(\mathbf{R S}_{n}\right)$ for $n>1$.

Theorem 9.1. Let $\mathbf{V} \in \mathcal{L}\left(\mathbf{R S}_{n}\right)$. Then $\mathbf{V}$ is exact if and only if any of the following holds:

(i) $A_{2} \in \mathbf{V}$;

(ii) $N_{1} \notin \mathbf{V}$;

(iii) $\mathbf{V} \in\left[\mathbf{B}_{\mathbf{2}}, \mathbf{N B}_{\mathbf{2}} \vee \mathbf{G}_{n}\right]$;

(iv) $B_{2}, M_{p} \in \mathbf{V}$ for some prime $p$ dividing $n, A_{0} \notin \mathbf{V}$. 
Proof. Let $\mathbf{V}$ be an exact variety. By Theorem 2.1, we know that either (i) or (ii) holds or

$$
B_{2} \in \mathbf{V}, \quad A_{0} \notin \mathbf{V}
$$

By Proposition 2.6, this translates to

$$
\mathbf{V} \in\left[\mathbf{B}_{\mathbf{2}},\left[x^{n} y^{n}=\left(x^{n} y^{n}\right)^{n+1}\right] \cap \mathbf{R S}_{n}\right]
$$

If (iii) does not hold, then by Theorem 8.2, $\mathbf{V}$ contains a variety of the form $\mathbf{B}_{\mathbf{2}} \vee \mathbf{M}_{p}$, so that (iv) holds. Thus one of the conditions (i)-(iv) must hold.

Conversely, if either (i) or (ii) holds, then $\mathbf{V}$ is exact by Theorem 2.1. If (iii) holds, then $\mathbf{V}$ is exact by Theorem 6.2 and Corollary 6.3. If (iv) holds, then $\mathbf{V}$ is exact by Theorem 8.1.

Let $\mathbf{V} \in \mathcal{L}\left(\mathbf{R S}_{n}\right)$ satisfy the condition that $N_{1} \notin \mathbf{V}$. By Theorem $2.1, \mathbf{V}$ is exact and generated by completely 0-simple semigroups. However, if $S=\mathcal{M}^{0}(I, G, \Lambda ; P) \in \mathbf{V}$ then there can be no zero entries in $P$, since otherwise $N_{1}$ would divide $S$. Hence the completely 0 -simple semigroups in $\mathbf{V}$ must be either completely simple or completely simple with a zero adjoined (the latter case including a two-element semilattice). Thus $\mathbf{V}$ is a variety of normal bands of groups of exponent dividing $n$. Let $\mathbf{N B G}_{n}$ be the variety of normal bands of groups of exponent dividing $n$. By Petrich-Reilly [8, Theorem IV.1.6], every subvariety of $\mathbf{N B G}_{n}$ is exact. Thus, for $\mathbf{V} \in \mathcal{L}\left(\mathbf{R S}_{n}\right)$,

$$
\mathbf{N}_{\mathbf{1}} \notin \mathbf{V} \Longleftrightarrow \mathbf{V} \in \mathcal{L}\left(\mathbf{N B G}_{n}\right)
$$

Let $p_{1}, p_{2}, \ldots, p_{m}$ denote the distinct prime divisors of $n>1$. Then, from Theorem 9.1 and the above remarks, we can conclude the following.

COROLlARY 9.2. The exact subvarieties of $\mathbf{R S}_{n}$ are precisely those varieties in the following intervals (see Figure 1):

$$
\begin{gathered}
{\left[\mathbf{T}, \mathbf{N B G}_{n}\right], \quad\left[\mathbf{B}_{\mathbf{2}}, \mathbf{N B}_{\mathbf{2}} \vee \mathbf{G}_{n}\right],} \\
{\left[\mathbf{B}_{\mathbf{2}} \vee \mathbf{M}_{p_{i}},\left[x^{n} y^{n}=\left(x^{n} y^{n}\right)^{n+1}\right] \cap \mathbf{R S}_{n}\right] \quad(i=1,2, \ldots, m),} \\
{\left[\mathbf{A}_{\mathbf{2}}^{*}, \mathbf{R S}_{n}\right] .}
\end{gathered}
$$

\section{Acknowledgements}

The author would like to thank E. W. H. Lee for many stimulating discussions. His discovery of a variety satisfying Kublanovsky's condition (3) in Theorem 2.1 that is not exact prompted this investigation. The author would also like to thank the anonymous referee for his many helpful comments. 


\section{References}

[1] R. L. Graham, 'On finite 0-simple semigroup and graph theory', Math. Systems Theory 2 (1968), 325-339.

[2] T. E. Hall, S. Kublanovsky, S. Margolis, M. Sapir and P. Trotter, 'Algorithmic problems for finite groups and finite 0-simple semigroups', J. Pure Appl. Algebra 119 (1997), 75-96.

[3] J. M. Howie, An Introduction to Semigroup Theory (Academic Press, London, 1976).

[4] S. I. Kublanovsky, On the Rees-Sushkevich variety, manuscript.

[5] S. I. Kublanovsky, E. W. H. Lee and N. R. Reilly, 'Sufficient conditions for the exactness of ReesSushkevich varieties', Semigroup Forum 76 (2008), 87-94.

[6] G. I. Mashevitzky, 'Identities in Brandt semigroups', in: Semigroup Varieties and Semigroups of Endomorphisms, Collect. Sci. Works (Leningrad Gos. Ped. Inst., Leningrad, 1979), pp. 126-137 (in Russian).

[7] G. I. Mashevitzky, 'Varieties generated by completely 0-simple semigroups', in: Semigroups and Their Homomorphisms (ed. E. S. Lyapin) (Ross. Gos. Ped. Univ., Leningrad, 1991), pp. 53-62 (in Russian).

[8] M. Petrich and N. R. Reilly, Completely Regular Semigroups (John Wiley and Sons, New York, 1999).

[9] N. R. Reilly, The interval $\left[\mathbf{B}_{2}, \mathbf{N B}_{2}\right]$ in the lattice of Rees-Sushkevich varieties, Algebra Universalis, to appear.

[10] N. R. Reilly, 'Complete congruences on the lattice of Rees-Sushkevich varieties', Comm. Algebra 35 (2007), 3624-3659.

[11] A. N. Trahtman, 'A basis of identities of a five-element Brandt semigroup', in: Issledovanija po Sovremennoj Algebre (Studies in Contemporary Algebra) (ed. L. N. Shevrin), Ural. Gos. Univ. Mat. Zap. 12, (3), 147-149, 1981 (in Russian) Sverdlovsk.

[12] A. N. Trahtman, 'Identities of a five-element 0-simple semigroup', Semigroup Forum 48 (1994), 385-387.

[13] M. V. Volkov, 'On a question by Edmond W. H. Lee', Izv. Ural. Gos. Univ. Mat. Mekh. 7(36) (2005), 167-178.

NORMAN R. REILLY, Department of Mathematics, Simon Fraser University, 8888 University Drive, Burnaby, BC, Canada V5A 1S6

e-mail: nreilly@cs.sfu.ca 